\title{
Complex Movement Control in a Rat Model of Parkinsonian Falls: Bidirectional Control by Striatal Cholinergic Interneurons
}

\author{
Cassandra Avila, ${ }^{*}{ }^{\mathbb{D}}$ Aaron Kucinski, ${ }^{*}$ and ${ }^{\circledR}$ Martin Sarter \\ Department of Psychology, University of Michigan, Ann Arbor, Michigan 48103
}

Older persons and, more severely, persons with Parkinson's disease (PD) exhibit gait dysfunction, postural instability and a propensity for falls. These dopamine (DA) replacement-resistant symptoms are associated with losses of basal forebrain and striatal cholinergic neurons, suggesting that falls reflect disruption of the corticostriatal transfer of movement-related cues and their striatal integration with movement sequencing. To advance a rodent model of the complex movement deficits of Parkinsonian fallers, here we first demonstrated that male and female rats with dual cortical cholinergic and striatal DA losses (DL rats) exhibit cued turning deficits, modeling the turning deficits seen in these patients. As striatal cholinergic interneurons (ChIs) are positioned to integrate movement cues with gait, and as $\mathrm{ChI}$ loss has been associated with falls in PD, we next used this task, as well as a previously established task used to reveal heightened fall rates in DL rats, to broadly test the role of ChIs. Chemogenetic inhibition of ChIs in otherwise intact male and female rats caused cued turning deficits and elevated fall rates. Spontaneous turning was unaffected. Furthermore, chemogenetic stimulation of ChIs in DL rats reduced fall rates and restored cued turning performance. Stimulation of ChIs was relatively more effective in rats with viral transfection spaces situated lateral to the DA depletion areas in the dorsomedial striatum. These results indicate that striatal ChIs are essential for the control of complex movements, and they suggest a therapeutic potential of stimulation of ChIs to restore gait and balance, and to prevent falls in PD.

Key words: acetylcholine; cortex; falls; interneurons; Parkinson's disease; striatum

Significance Statement

In persons with Parkinson's disease, gait dysfunction and the associated risk for falls do not benefit from dopamine replacement therapy and often result in long-term hospitalization and nursing home placement. Here, we first validated a new task to demonstrate impairments in cued turning behavior in rodents modeling the cholinergic-dopaminergic losses observed in Parkinsonian fallers. We then demonstrated the essential role of striatal cholinergic interneurons for turning behavior as well as for traversing dynamic surfaces and avoiding falls. Stimulation of these interneurons in the rat model rescued turning performance and reduced fall rates. Our findings indicate the feasibility of investigating the neuronal circuitry underling complex movement control in rodents, and that striatal cholinergic interneurons are an essential node of such circuitry.

\section{Introduction}

Stepping onto stairs or onto an escalator, turning, or circumventing an obstacle involve the disruption of the regular patterning of

Received Jan. 28, 2020; revised May 10, 2020; accepted May 15, 2020.

Author contributions: C.A. and M.S. designed research; C.A. and A.K. performed research; C.A. and A.K. analyzed data; C.A., A.K. and M.S. wrote the paper.

${ }^{*}$ C.A. and A.K. contributed equally to this work.

The authors declare no competing financial interests.

This work was supported by National Institutes of Health Grant P50-NS-091856 (to the Morris K. Udall Center for Excellence in Parkinson's Disease Research). We thank Drs. R. Albin and N. Bohnen (University of

Michigan) for comments on an earlier version of the manuscript.

Correspondence should be addressed to Martin Sarter at msarter@umich.edu.

https://doi.org/10.1523/JNEUROSCI.0220-20.2020

Copyright $\odot 2020$ the authors movements and often require corrective actions to counter postural and stepping errors. In such situations, the attentional "spotlight" shifts toward, and prioritizes, the processing of movement-related information to guide corrective movements (Brown et al., 2002; Doumas et al., 2008; Yogev-Seligmann et al., 2012; Mersmann et al., 2013). In older persons and persons with Parkinson's disease (PD), limitations in attentional capacities predict complex movement control deficits (Allcock et al., 2009; Lord et al., 2010; O'Halloran et al., 2011; Yarnall et al., 2011; Montero-Odasso et al., 2012; Amboni et al., 2013; Nagamatsu et al., 2013; Stuart et al., 2017).

In PD, impaired gait, postural instability and falls are resistant to dopamine (DA) replacement therapy (Bloem et al., 2003; Grimbergen et al., 2004). Positron emission topography (PET) studies indicated that these symptoms are associated with a 
reduced cholinergic innervation of the cortex and thalamus (Bohnen et al., 2009b, 2012, 2013; Müller et al., 2013), and with a reduced expression of the vesicular acetylcholine transporter (VAChT) in the striatum, the latter presumably reflecting loss of cholinergic interneurons (ChIs; Bohnen et al., 2019; for additional evidence linking cholinergic losses with falls, see Rochester et al., 2012). Consistent with evidence indicating that cholinergic signaling is essential for the detection of cues in attentional contexts (McGaughy et al., 1996; Dalley et al., 2004; St Peters et al., 2011; Gritton et al., 2016; Sarter et al., 2016; Berry et al., 2017; Howe et al., 2017; Kim et al., 2019; Sarter and Lustig, 2020), cholinergic losses are thought to disrupt the cortical processing of task and movement cues, thereby depriving the striatum of information to guide (corrective) movements (Sarter et al., 2014). In interaction with the PD-defining striatal DA depletion, the selection and filtering of cortical inputs is further impaired (Strafella et al., 2005; Guthrie et al., 2013); yielding gait dysfunction and an elevated risk for falls (but see Emmons et al., 2017; Hart et al., 2018a,b).

ChIs are the target of cortical as well as thalamic striatal afferents (Guo et al., 2015; Klug et al., 2018; Mamaligas et al., 2019). Acetylcholine (ACh) released from ChIs controls glutamate signaling from these afferents and inhibits output neurons (Zucca et al., 2018), suggesting that Chls select corticostriatal inputs to guide movement shifts and corrections (Doig et al., 2014). Interactions between ChIs, corticostriatal, thalamostriatal, and nigrostriatal afferents are reciprocal (Threlfell et al., 2012; Chuhma et al., 2014; Maurice et al., 2015; Kosillo et al., 2016); these interactions position ChIs as an essential striatal integrator orchestrating complex movements and error-triggered corrections (Apicella, 2017; Aoki et al., 2018; Tanimura et al., 2018; Gritton et al., 2019).

We previously established a rat model [dual lesioned (DL) rats] of the combined cortical cholinergic and striatal DA losses observed in Parkinsonian fallers. DL rats exhibit high fall rates while traversing highly dynamic surfaces, as measured by the Michigan complex movement control task (MCMCT). Fall rates were correlated with impairments in attentional performance. Cholinergic or DA losses alone did not increase fall rate (Kucinski et al., 2013, 2015; Sarter et al., 2014; Kucinski and Sarter, 2015). Here, we first describe a new task, the cue-triggered turning task (CTTT), to broaden and specify the demonstration of complex movement deficits in rodents. The ability of DL rats to execute cued turns was disrupted, mirroring turning deficits observed in Parkinsonian fallers (Stack and Ashburn, 2008; Cheng et al., 2014). We then demonstrated that chemogenetic inhibition of ChIs in the dorsomedial striatum is sufficient to cause turning deficits and high fall rates in otherwise intact rats, and that stimulation of ChIs reduces falls and restores cued turning performance in DL rats.

\section{Materials and Methods}

Subjects

Data from a total of $N=90$ rats ( 67 females) formed the basis for the evidence obtained from the present experiments. For the determination of CTTT performance by DL rats and sham-lesioned controls $(n=32,19$ females) Sprague Dawley rats (Envigo) were used. Animals were 36 months of age at the beginning of the experiment. For experiments involving designer receptors exclusively activated by designer drugs (DREADDs), ChAT-Cre heterozygous Long-Evans male rats were obtained from Stanford University (Department of Bioengineering) and bred with wild-type (WT) Long-Evans female rats obtained from Envigo. Tail snips were used for genotyping by Transnetyx. A total of $n=56$ female ChAT-Cre rats, between 2 and 3 months of age at the beginning of experiments, were used to assess the effects of DREADD activation on CTTT and MCMCT performance. The effects of DREADD-mediated inhibition of ChIs were determined in otherwise intact rats (hM4Di rats: $n=19,14$ females; mCherry-expressing controls: $n=17,14$ females). DREADD-mediated stimulation of ChIs (hM3Dq rats) was determined in DL ( $n=10$ females) and sham-operated control rats ( $n=9$ females). An additional eight female DL rats expressing the non-DREADD control construct were used to assess potential off-target effects of clozapine- $\mathrm{N}$-oxide (CNO). Of the 36 rats expressing the inhibitory DREADD and the control construct, 31 rats were also tested 2 weeks earlier on the MCMCT; an additional 10 rats were prepared for subsequent CTTT testing. Tables 1 and 2 describe presurgery and postsurgery CTTT and MCMCT training and testing. Table 3 provides a sequence of events and timeline for the experiment involving the expression and effects of activation of an excitatory DREADD in DL rats and shamoperated controls on MCMCT and CTTT performance.

Rats were individually housed in opaque single standard cages $(27.70 \times 20.30 \mathrm{~cm})$ in a temperature- and humidity-controlled environment $\left(23^{\circ} \mathrm{C}, 45 \%\right)$ and maintained under a $12 \mathrm{~h}$ light/dark schedule (lights on at 7:00 A.M.). Food (Envigo Teklad rodent diet) and water were available ad libitum. MCMCT and CTTT testing were conducted during the light phase (7:00 A.M. to 7:00 P.M.). All procedures were conducted in adherence with protocols approved by the Institutional Animal Care and Use Committee of the University of Michigan and in laboratories accredited by the Association for Assessment and Accreditation of Laboratory Animal Care.

\section{CTTT}

The treadmill (see Fig. $2 a$ ), $32 \mathrm{~cm}$ wide by $90 \mathrm{~cm}$ long, was adapted from a 2200 Series Flat Belt End Drive Dorner conveyer. The conveyer belt surface material was polypropylene, friction resistant, and easily cleaned with ethanol or soap. The conveyer was paired with a Dorner Variable Speed Controller, with speeds ranging from 0 to $32 \mathrm{~cm} / \mathrm{s}(19.2 \mathrm{~m} / \mathrm{min})$. The Variable Speed Controller included a reversing controller, which allowed for the belt to rotate clockwise (cw), "forward," or counterclockwise (cc), "reverse." A Faraday cage made in-house was placed on top of this conveyer, $54.61 \mathrm{~m}$ in length, $31.75 \mathrm{~m}$ wide, and $36.83 \mathrm{~m}$ tall. The wooden frame of the Faraday cage was enclosed by a woven copper mesh and grounded to block out static electric fields. Plexiglas inserts were placed inside the Faraday cage to prevent rodents from chewing on the mesh. This shielding is used in other ongoing experiments. Experimenters raised two wooden panels on top of the cage to place rodents on the treadmill. The $28 \mathrm{~V} \mathrm{DC}, 100 \mathrm{~mA}$ stimulus light was $\sim 2.54 \mathrm{~cm}$ in diameter with a flat lens and mounted one side lengthwise on the Faraday cage (Med Associates). The auditory cue was a Mallory Sonalert audible device. This $28 \mathrm{~V} \mathrm{DC}, 18 \mathrm{~mA}$ device was mounted on the opposite side to the visual cue and was $\sim 4.3 \mathrm{~cm}$ long by $4.3 \mathrm{~cm}$ wide by $3.6 \mathrm{~cm}$ tall. This device emitted a continuous tone at a sound pressure level of $68 \mathrm{dBA}$ (decibels using A-weighted sound levels), which is within the acceptable range for chronic presentations to neither elicit a fearful response nor induce hearing loss (Turner et al., 2005; Castelhano-Carlos and Baumans, 2009). These devices were controlled by a Med Associates interface and a relay module to run our custom program. Briefly, rats were trained to walk on a treadmill until the onset of a "stop" or a "turn" cue, following which the treadmill restarted in the same or the reverse direction, respectively. As illustrated in Figure $2 a$, the cue is presented for $2 \mathrm{~s}$, and $1 \mathrm{~s}$ later the treadmill pauses for $5 \mathrm{~s}$. Rats learn to respond to one cue to stop and the other cue to turn, then the treadmill restarts in the same or opposite direction.

Performance sessions were videotaped with four cameras [resolution, 600 television lines; scanning frequency, $15.734 \mathrm{kHz}(\mathrm{H})$ and $59.94 \mathrm{~Hz}$ $(\mathrm{V})$; pixels $795(\mathrm{H}) \times 596(\mathrm{~V})$; power, DC $12 \mathrm{~V}( \pm 10 \%)$; maximal, $80 \mathrm{~mA}$; model KPCS190SH Black/White Bullet Camera with one-third inch SONY Super HAD CCD, KT\&C] mounted outside of and at each side of the Faraday cage. The images were relayed to four grids by a splitter (Q8700 Digital Quad Unit B\&H, Clover) and displayed simultaneously on a monitor (CT-1384-V, Panasonic). Sessions were recorded using a DVD-R (DR430, Toshiba) and Verbatim DVRs, and the 
Table 1. CTTT training and testing conditions

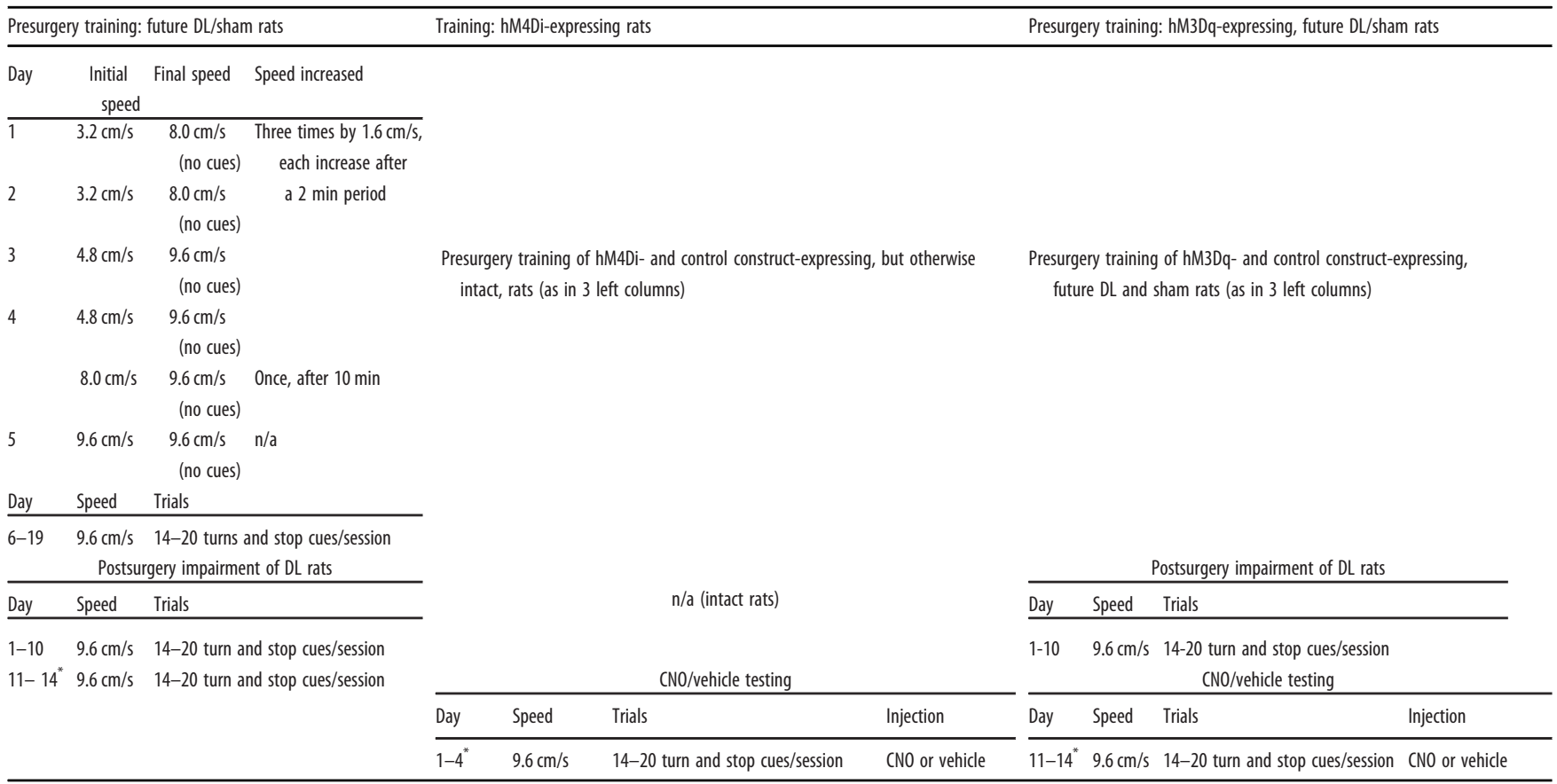

* Data used for analysis. n/a, Not applicable.

Table 2. MCMCT Sequence of testing conditions and timeline for excitatory DREADD activation in DL rats on MCMCT and CTTT performance

\begin{tabular}{llll}
\hline Day & Beam & $\begin{array}{l}\text { Rotating } \\
\text { speed }(\mathrm{rpm})\end{array}$ & Trials $(n)$ \\
\hline & \multicolumn{1}{c}{ Presurgery } & & \\
1 & Plank & $\mathrm{n} / \mathrm{a}$ & 6 \\
2 & Straight rod & $0 \mathrm{rpm}$ & 6 \\
3 & cc rotating rod & $5 \mathrm{rpm}$ & 6 \\
\hline
\end{tabular}

Postsurgery: inhibitory DREADD in intact rats, and excitatory DREADD in DL rats

1 (vehicle)

Plank $\quad n / a$

Straight rod $\quad 0 \mathrm{rpm}$

2 (CNO or vehicle)

3 (CNO or vehicle)

cc rotating rod

$8 \mathrm{rpm}$

cc rotating rod

$8 \mathrm{rpm}$

cW rotating rod

cw rotating rod

$8 \mathrm{rpm}$

$8 \mathrm{rpm}$

5 (CNO or vehicle)

recordings were converted to MPEG-4 Part 14 (mp4) using VLC media software [version 3.0.6 (free open source)].

Training regimen. Rats underwent a treadmill acclimation regimen that was adapted from Arnold and Salvatore (2014). Rats were handled for $20 \mathrm{~min}$ for $2 \mathrm{~d}$ before exposure to the treadmill. On the second day, rats were placed in the Faraday cage for $30 \mathrm{~min}$ with the belt paused. To minimize stressors, as well as to increase the discriminability of the light cue, all sessions were conducted under dim red lighting. For a week, rats were first placed into the Faraday cage for $3 \mathrm{~min}$ with the belt paused and then trained to walk on the treadmill at speeds up to $9.6 \mathrm{~cm} / \mathrm{s}$ or $\sim 6$ $\mathrm{m} / \mathrm{min}$. The initial speed and direction of the treadmill were manually set by the experimenter, with initial direction counterbalanced within and across subjects. During this training, rats were reoriented by gentle prodding if they began to walk in the opposite direction of the belt. Upon each session completion, rats were immediately returned to their home cages and housing room. Between sessions and subjects, the Plexiglas walls of the Faraday cage and the treadmill belt were wiped down with $70 \%$ ethanol and soap.

For the first $2 \mathrm{~d}$, the initial treadmill speed was set at $3.2 \mathrm{~cm} / \mathrm{s}$ and gradually increased by $1.6 \mathrm{~cm}$ every $2 \mathrm{~min}$, up to a maximum speed of
Table 3. Timeline for excitatory DREADD activation in DL rats on MCMCT and CTTT performance

\begin{tabular}{ll}
\hline Duration (d) & \multicolumn{1}{c}{ Procedure } \\
\hline 3 & MCMCT presurgery training \\
First surgery & Striatal DREADD infusions \\
21 & Postsurgery recovery \\
19 & CTT prelesion training \\
Second surgery & Neurotoxin infusions into the basal forebrain and striatum \\
7 & Postsurgery recovery \\
1 & MCMCT stationary rod testing (vehicle) \\
4 & MCMCT rotating rod testing (vehicle or CNO) \\
7 & Home cage \\
10 & CTTT retraining \\
4 & CTTT testing (vehicle or CNO) \\
\hline
\end{tabular}

$8 \mathrm{~cm} / \mathrm{s}$. Each session lasted $20 \mathrm{~min}$. This initial maximum speed was selected to allow the rats to habituate before running at a faster speed to reduce attrition due to noncompliance. On day 3, the maximum speed was further increased to $9.6 \mathrm{~cm} / \mathrm{s}$. During testing days $3-5$, initial speeds increased (Table 1) until, on day 6 and thereafter, rats walked at $9.6 \mathrm{~cm} / \mathrm{s}$ for the full $20 \mathrm{~min}$. In the next phase of training (days 6-19) rats were placed on the treadmill and presented either an auditory or a visual cue. Contrary to the acclimation training phase, when experimenters manually controlled the treadmill, this phase was completely controlled by Med-PC software and interface (Med Associates). The cues were presented for $2 \mathrm{~s}$, and a cue of one modality was presented at a maximum twice in a row. The mean intertrial interval (ITI) was $45 \pm 30 \mathrm{~s}$ (see Fig. $2 a$, illustration of the main task events). The total number of trials per daily session was $14-20$, with a maximum session duration of $15 \mathrm{~min}$. Rats underwent cue training for $\sim 2$ weeks and were passed onto the testing phase on reaching performance criterion, defined as $70 \%$ correct responses to both cues, and that these responses occurred before the restart of the treadmill belt for 2 consecutive days. For assessment of the effects of $\mathrm{CNO}$, rats received either vehicle injections one day and $\mathrm{CNO}$ (50 min before session onset) the other day. CNO or vehicle was administered twice, and test day was a factor in the analysis (see Administration of $\mathrm{CNO}$ ).

CTTT performance measures. Performance measures (turns, missed turns, stops, no stops) were extracted from offline scoring of session 
videos. For a successful turn to be scored, the animal must have initiated a turn, defined by a rotation of the longitudinal orientation of the body of at least $90^{\circ}$, before the treadmill restart, that is, within $8 \mathrm{~s}$ of the cue onset (see Fig. 2a). A stop was defined as a cessation of forward movement. For stops occurring before the treadmill stop, rats typically stopped while positioned at the back end of the treadmill, so that, following such a stop, the treadmill transported them to the front of the treadmill (within the remaining 1-2 s until the treadmill stopped), and without the rat contacting the front end of the test chamber. Following the treadmill stop, and during the $5 \mathrm{~s}$ pause, a stop failure was scored if the animal (falsely) turned. Turns and stops were expressed as a ratio of the number of trials per session that began with turn or stop cues, respectively. Furthermore, the proportion of cued responses executed during the period beginning with the cue onset and until the treadmill stopped were separately analyzed to obtain a measure reflecting immediate, cue-evoked responses.

\section{Open field turns}

The main purpose of assessing the effects of CNO in DREADD-expressing rats exploring an open field was to determine whether activation of the DREADD, or the effects of CNO per se, interfered with the spontaneous turning preferences of the rats. If such an effect of $\mathrm{CNO}$ were found, the effects of $\mathrm{CNO}$ on cued turns in hM4Di-expressing rats tested in the CTTT may have been confounded by effects that interfered with their preferred turning direction. The open field box was constructed of Plexiglas $(61.00 \mathrm{~cm}$ long and wide, and $30.00 \mathrm{~cm}$ high) and separated into five quadrants. The four peripheral quadrants were sized $30.25 \mathrm{~cm}^{2}$ minus the overlapping quarter of the central quadrant $\left(12.75 \mathrm{~cm}^{2}\right)$, and the central quadrant was $25.50 \mathrm{~cm}^{2}$ in size. A Canon VIXIA HF R800 Camcorder was mounted on a Magnus PV-3330 Photograph Tripod with a three-way pan and tilt head and positioned at a $90^{\circ}$ angle directly above the box during testing for recordings. Rats were injected $50 \mathrm{~min}$ before placement into the central quadrant. The effects of the two injections were assessed on consecutive days. The order of injections (vehicle vs $\mathrm{CNO}$ ) was counterbalanced among rats. Rats could freely explore the box for $3 \mathrm{~min}$. This duration was chosen based on pilot data suggesting that rats would continue exploring the open field a day later when the first exposure was limited to this period. The sessions were run under fluorescent lighting and the box was wiped down with $70 \%$ ethanol after each rat was tested. We determined the number of right and left turns, defined as a rotation of the longitudinal orientation of the body of at least $90^{\circ}$ clockwise or counterclockwise. The relative time spent in the central versus peripheral quadrants was quantified offline by undergraduate research assistants (URAs) blind to the treatment condition. Time spent was calculated by marking the time an animal was determined to have entered a quadrant (when three-quarters of their upper body was positioned in the quadrant) to the time it took for an animal to exit a quadrant (when they were determined to have entered a new quadrant). The total time spent in the central quadrant was then compared against the total time spent in the peripheral quadrants (summed together). Moreover, average locomotor speed (in millimeters per second), and total distance traveled (in millimeters) were determined using ToxTrac, an open source animal-tracking software (Rodriguez et al., 2018).

\section{MCMCT}

The MCMCT beam traversal apparatus (Kucinski et al., 2013, 2018, 2019; Sarter et al., 2014; Koshy Cherian et al., 2019) assesses the ability of rats to perform attention-demanding beam traversals and correct for stepping errors while crossing a narrow square rod surface (side length, $1.59 \mathrm{~cm}$; see Fig. $4 a$ ). Traversal of the rod, particularly when rotating, reliably caused falls in DL rats (Kucinski et al., 2013; Kucinski and Sarter, 2015). Recently, we designed and validated a second MCMCT apparatus that allows testing of additional complex traversal surfaces (Kucinski et al., 2018). This apparatus features longer $(3.0 \mathrm{~m})$ square rods (side length, $1.59 \mathrm{~cm}$ ), composed of aluminum tubing covered with gray gaffer's tape for traction. There was also a plank surface (width, $13.3 \mathrm{~cm}$ ) that could be placed directly on top of the straight rod and fitted firmly in place inside edges of the support towers. This surface was used to familiarize rats with traversing from goal box to goal box. The rods were rotated using a $12 \mathrm{~V}$ DC electric motor controlled remotely by a pulse width modulator that was able to adjust the speed of rotation (up to $10 \mathrm{rpm}$ ) and switch the direction of rotation. A safety net was suspended $20 \mathrm{~cm}$ under the beam to catch the rats during falls. Two identical end stations were situated on top of the support towers at opposite ends of the beam. These stations consisted of a $30 \times 25 \mathrm{~cm}$ platform with a 3 -cm-diameter copper cup embedded in the floors. Rats were given a banana pellet ( $45 \mathrm{mg} /$ pellet; BioServ) placed inside the cups in the goal boxes following each traversal. Each goal box was surrounded by a retractable wall structure $(23 \mathrm{~cm}$ height in raised position) to allow conversion from an open platform to a boxed structure. On the wall facing the beam of each box structure a $9-\mathrm{cm}$-wide opening allowed the rats access to and for the beam. The walls were raised and lowered mechanically with a $12 \mathrm{~V}$ DC electric motor actuated remotely by a toggle switch. Lowering the walls and thereby turning the box into an open field platform was sufficient for rats to initiate beam traversal to enter the opposite box (with walls up) and consume the banana pellet. Between traversals, the walls of the goal box remained in the up position for $45 \mathrm{~s}$. Videos of traversals were recorded with four bullet Marshall 1080-HDDI model CV500 Series cameras (B-30/25P frame rate/59.94i) mounted on the net frame parallel to one side of the beam. The videos were converted to a single feed using a quad SDI-to-HDMI multiviewer (MicroQuad, Matrox) and viewed directly on a PC using Elgato Game Capture HD software.

In experiments testing the effects of activation of hM4Di or hM3Dq DREADDs, rats were first habituated with a $3 \mathrm{~d}$ traversal sequence, including trials on the plank, and stationary and rotating (straight) rods (Table 2). Following surgery and recovery, the effects of CNO were assessed. All animals received vehicle injections before performing six runs on day 1 -three on the plank surface and three on the stationary rod. Rats were then tested on two traversal conditions, each for 2 consecutive days-the cc rotating rod ( $8 \mathrm{rpm}$ ) on test days 2 and 3 , and the $\mathrm{cw}$ rotating rod $(8 \mathrm{rpm})$ on test days 4 and 5 (Table 2 ).

Before testing the effects of activation of the inhibitory hM4Di DREADD, rats were randomly assigned to one of two test cohorts. The first cohort ( $n=6$ expressing the inhibitory hM4Di DREADD; $n=7$ expressing the mCherry-expressing control construct) received vehicle injections on days 2 and 4, and CNO on days 3 and 5. The second cohort ( $n=6$ expressing the inhibitory DREADD; $n=6$ expressing the mCherryexpressing control construct) received $\mathrm{CNO}$ on days 2 and 4 and vehicle on days 3 and 5. Injections of $\mathrm{CNO}$ or vehicle were given $30-60 \mathrm{~min}$ before MCMCT testing (drug preparation details below). To assess the excitatory hM3Dq DREADD in DL and sham rats, animals were similarly randomly grouped into one cohort that received vehicle on days 2 and 4 ( $n=5 \mathrm{DL} ; n=4$ shams), and one cohort that received vehicle on days 3 and 5 ( $n=5 \mathrm{DL} ; n=3$ shams).

\section{MCMCT performance measures}

Falls, slips, and traversal time were analyzed in the same manner as previously described (Kucinski et al., 2013, 2017), with the exception that the performance of the rats over each entire traversal was considered, due to the longer length of the rods when compared with earlier experiments. Rats were allowed to fall into the net and placed back onto the rod or, on an imminent fall, the experimenter assisted the rat in regaining balance on the rod, and no further slips or falls were counted until the rat regained a balanced posture and resumed forward movement. In either case, traversal time was corrected for fall-related disruptions of forward movement. In the overwhelming number of cases, rats were able to regain forward movement within $2 \mathrm{~s}$ of being placed back onto the rod. Because multiple falls could occur during a run, falls per run were used as a metric for fall rates.

Video-based analysis of CTTT and MCMCT performance: blinding procedures and inter-rater reliability

CTTT-derived performance measures (turns, missed turns, stops, no stops) were extracted from session videos scored offline by blinded URAs. Blinding was further maintained by requiring that an individual URA only scored the behavior of animals tested by another URA. Identifiers were extracted following the completion of scorings. Interrater reliability scores were computed for scoring turns and stops during 
a total of 60 randomly selected trials of rats from all groups and treatments (Cohen's $\kappa=0.92$ and 0.97, respectively). MCMCT-derived performance measures were similarly scored offline by two scorers blinded to testing conditions (group and treatment). Based on a sample of a total of 36 runs on the MCMCT, selected from six rats, the two raters were in "almost perfect" (Landis and Koch, 1977) agreement (Cohen's $\kappa=0.83$ ).

\section{Neurotoxin infusion surgery}

To demonstrate that the CTTT reveals deficits that parallel the falls seen in DL rats tested in the MCMCT (Kucinski et al., 2013), Sprague Dawley rats were anesthetized with isoflurane gas (induction, 4-5\%; maintenance, 2-3\%) delivered at $0.6 \mathrm{~L} / \mathrm{min} \mathrm{O}_{2}$ using a SurgiVet Isotec 4 Anesthesia Vaporizer and mounted to a stereotaxic instrument (David Kopf Instruments). The temperature of the animals was maintained at $37^{\circ} \mathrm{C}$ using Deltaphase isothermal pads (Braintree Scientific). Ophthalmic ointment was provided for lubrication of the eyes. A subcutaneous injection of $1 \mathrm{ml} / 100 \mathrm{~g} 0.9 \% \mathrm{NaCl}$ was administered to the animals to prevent hypovolemia and hemodynamic instability. Before surgery, rats also received carprofen $(5 \mathrm{mg} / \mathrm{kg}$, s.c.; Henry Schein Medical) for pain relief. Rats received bilateral bolus infusions $(4.0 \mu \mathrm{g} / \mu \mathrm{l}$; $1 \mu \mathrm{l} /$ hemisphere) of the neurotoxin 6-hydoxydopamine (Sigma-Aldrich; $n=16)$ or vehicle $(1 \mathrm{mg} / \mathrm{ml}$ ascorbic acid in $0.9 \% \mathrm{NaCl} ; n=9)$ into two sites of the striatum [from bregma: anteroposterior (AP), +1.2 or +0.2 $\mathrm{mm}$; mediolateral (ML), \pm 2.5 or $\pm 3.0 \mathrm{~mm}$; dorsoventral (DV), -5.0 or $-5.5 \mathrm{~mm}$ from dura]. Rats were injected with desipramine hydrochloride $(10 \mathrm{mg} / \mathrm{kg}$, i.p.; Sigma-Aldrich) $30 \mathrm{~min}$ before neurotoxin infusions for the protection of noradrenergic neurons (Breese and Traylor, 1971). These rats also received bilateral infusions $(200 \mathrm{ng} / \mu \mathrm{l} ; 0.8 \mu \mathrm{l} / \mathrm{hemisphere})$ of the cholino-specific immunotoxin 192 IgG-Saporin (Advanced Targeting Systems; $n=16)$ or ACSF $(n=9)$ into the nucleus basalis and substantia innominata of the basal forebrain (from bregma: AP, -0.8 $\mathrm{mm}$; ML, $\pm 2.9 \mathrm{~mm}$; DV,$-7.8 \mathrm{~mm}$ ). An additional $8 \mathrm{~min}$ was allowed for diffusion for all infusions before the needle was removed. Nonabsorbable nylon sutures were used to close the incision. A topical antibiotic (Neosporin) was applied to the wound. Rats received one subsequent dose of carprofen $(5 \mathrm{mg} / \mathrm{kg}) 1 \mathrm{~d}$ postsurgery, followed by a 2 week recovery period before the initiation of behavioral testing. For experiments in DL rats also expressing the excitatory hM3Dq DREADD, the neurotoxins were infused in a separate, second surgery (Table 3, timeline).

\section{DREADD virus expression and CNO administration}

The viral vectors containing Cre-dependent plasmid pAAV-hSyn-DIOhM4D(Gi)-mCherry (catalog \#44 362-AAV8; titer of $2.1 \times 10^{13}$ genome copies (GC)/ml) or Cre-dependent control plasmid pAAV-hSyn-DIOmCherry (catalog \#50459-AAV8; titer of $2.2 \times 10^{13} \mathrm{GC} / \mathrm{ml}$ ) were obtained from AddGene. The adeno-associated virus (AAV), containing a double-floxed muscarinic $\mathrm{G}_{\mathrm{i}}$-coupled receptor, hM4D (Armbruster et al., 2007; Roth, 2016), fused with mCherry and under the control of human synapsin promoter, was stereotaxically infused bilaterally into the dorsomedial striatum. For the excitatory DREADD experiment, the Cre-dependent plasmid pAAV-hSyn-DIO-hM3D(Gq)-mCherry (cata$\log$ \#44361-AAV8, AddGene; titer of $4.0 \times 10^{12} \mathrm{GC} / \mathrm{ml}$ ) was infused into the dorsomedial striatum of DL and sham rats 2 weeks before the infusions of the neurotoxins or sham lesion surgeries. Rats were placed in vaporization chambers and anesthetized with $4-5 \%$ isoflurane delivered at $0.6 \mathrm{~L} / \mathrm{min} \mathrm{O}_{2}$ using a SurgiVet Isotec 4 Anesthesia Vaporizer until the animals were no longer responsive to a tail pinch and exhibited no hindlimb withdrawal reflex. The heads of the rats were shaved using electric clippers and cleaned with a betadine scrub and alcohol pad. The animals were then mounted to a stereotaxic instrument (David Kopf Instruments), and isoflurane anesthesia was maintained at $1-3 \%$ for the duration of the surgery. An $\sim 2.5 \mathrm{~cm}$ incision was made down the midline of the scalp to expose the skull. The body temperature of the animals was maintained at $37^{\circ} \mathrm{C}$ using Deltaphase Isothermal Pads (Braintree Scientific). Ophthalmic ointment was used for lubrication of the eyes. To prevent hypovolemia and hemodynamic instability during prolonged surgeries, $1 \mathrm{ml} / 100 \mathrm{~g} 0.9 \% \mathrm{NaCl}$ was administered subcutaneously. Animals also received an injection of an analgesic (carprofen; $5.0 \mathrm{mg} / \mathrm{kg}$, s.c) before surgery and once or twice daily as necessary for $48 \mathrm{~h}$ postoperatively. One microliter of AAV-hSyn-DIO-hM4D(Gi)-mCherry, AAV-hSyn-DIO$\mathrm{hM} 3 \mathrm{D}(\mathrm{Gq})$-mCherry, or AAV-hSyn-DIO-mCherry (control) vector was infused (bolus) into the dorsomedial striatum at two sites per hemisphere (site 1: AP, $+1.2 \mathrm{~mm}$; ML, $\pm 2.2 \mathrm{~mm}$; DV, $-4.5 \mathrm{~mm}$; site $2: \mathrm{AP},+0.2$ $\mathrm{mm}$; ML, $\pm 2.5 \mathrm{~mm}$; DV,$-4.5 \mathrm{~mm})$. The injector was left in place for $8 \mathrm{~min}$ to minimize diffusion into the injector tract.

\section{Administration of $\mathrm{CNO}$}

$\mathrm{CNO}$ was obtained from Tocris Bioscience and dissolved in $10 \mathrm{mg} / \mathrm{ml}$ in $6 \% \mathrm{DMSO}$ in $0.9 \% \mathrm{NaCl}$ solution. Injections of $\mathrm{CNO}$ or vehicle were administered with intraperitoneal injections at a dose of $5.0 \mathrm{mg} / \mathrm{kg} 30-60$ min before MCMCT or CTTT testing. To control for potential off-target effects of clozapine (Gomez et al., 2017), CNO was also administered to rats expressing a non-DREADD, mCherry-positive control construct. We previously did not find effects of this dose of CNO in control construct-expressing rats performing the MCMCT (Kucinski et al., 2019), consistent with the proposed conversion rate of $\mathrm{CNO}$ to clozapine (Gomez et al., 2017), yielding an equivalent clozapine dose of $0.05 \mathrm{mg} /$ $\mathrm{kg}$, and that a 50 - to 100 -fold higher dose of clozapine is required to produce direct effects on brain neurochemistry and numerous trained behaviors (Parada et al., 1997; Gemperle et al., 2003; Terry et al., 2003; Rueter et al., 2004; Martinez and Sarter, 2008; MacLaren et al., 2016; Smith et al., 2016; Mahler and Aston-Jones, 2018). Rats received either vehicle injections one day and CNO the other day (Tables 1,2). CNO or vehicle was administered twice, and test day was a factor in the initial analysis.

Visualization and quantification of the cholinergic-dopaminergic losses in $D L$ rats

Following the completion of the behavioral testing, rats were deeply anesthetized with a lethal dose of sodium pentobarbital $(270 \mathrm{mg} / \mathrm{kg}$, i.p.) and transcardially perfused with PBS followed by $4 \%$ paraformaldehyde in $0.15 \mathrm{~m}$ sodium phosphate solution, $\mathrm{pH}$ 7.4. Brains were extracted and postfixed in $4 \%$ paraformaldehyde for $24 \mathrm{~h}$, rinsed with PBS, and then placed in $30 \%$ sucrose solution until they sank. Brains were sectioned into $35-\mu \mathrm{m}$-thick slices using a freezing microtome (model CM 2000R, Leica) and stored in cryoprotectant until further histologic processing. Sections from lesioned and sham-lesioned rats were stained, separately, for the visualization of acetylcholinesterase (AChE)-positive fibers in the cortex and the presence of striatal tyrosine hydroxylase (TH). AChE visualization was performed using a protocol adopted from Tago et al. (1986). Sections were first rinsed three times for $5 \mathrm{~min}$ each in $0.1 \mathrm{M}$ phosphate buffer, $\mathrm{pH} 7.2$, then incubated in $0.1 \%$ hydrogen peroxide [diluted from $30 \%(\mathrm{w} / \mathrm{w})$ in $\mathrm{H}_{2} \mathrm{O}$ solution; Sigma Aldrich] for $30 \mathrm{~min}$. The sections were then rinsed with maleate buffer three times for $5 \mathrm{~min}$ each and incubated in maleate buffer $(200 \mathrm{ml} ; \mathrm{pH} 6.0)$, containing acetylthiocholine iodide $(6 \mathrm{mg}), 0.1 \mathrm{M}$ sodium citrate $(0.5 \mathrm{ml}), 30 \mathrm{~mm}$ cupric sulfate $(1.0 \mathrm{ml})$, and $5.0 \mathrm{~mm}$ potassium ferricyanide $(1.0 \mathrm{ml})$ for $75 \mathrm{~min}$. Sections were then rinsed three times for $5 \mathrm{~min}$ each in $30 \mathrm{~mm}$ Tris buffer $(250 \mathrm{ml}), \mathrm{pH} 7.6$, and incubated in $30 \mathrm{~mm}$ Tris buffer $(250 \mathrm{ml})$ containing ammonium nickel sulfate $(0.75 \mathrm{~g})$, and diaminobenzidine (DAB; $100 \mathrm{mg}$; final $\mathrm{pH}$ 6.2). Following this, hydrogen peroxide (30\%; $8.0 \mu \mathrm{l} / 40 \mathrm{ml}$ solutions) was added, and the sections were last rinsed three times for $5 \mathrm{~min}$ each with $3 \mathrm{~mm}$ Tris buffer. TH immunostaining was performed using a primary antibody (ab152; Millipore Sigma; rabbit anti-TH) and Vectastain Elite ABC kit (PK-6100, Vector Laboratories). Sections were first rinsed nine times with $0.1 \mathrm{M}$ phosphate Tris buffer solution (PBT), $\mathrm{pH} 7.4$, for 5 min each and incubated in $1 \% \mathrm{H}_{2} \mathrm{O}_{2}$ for 30 min. Sections were then rinsed three times with PBT for $10 \mathrm{~min}$ and incubated in the primary antibody [rabbit anti-TH made in 3\% normal donkey serum (NDS), 1:500] overnight. The following day, the sections were rinsed three times with PBT for 10 min each and incubated in the secondary antibody (705-065-147; Jackson ImmunoResearch; biotinylated donkey anti-rabbit 1:500 made in 1\% NDS). Sections were then rinsed three times with $\mathrm{PBT}$ for $5 \mathrm{~min}$ and incubated in $\mathrm{ABC}$ Elite (1:1000) for $60 \mathrm{~min}$ at room temperature. After, they were rinsed three times for 5 min each in PBT, developed for 8 min using a DAB substrate solution, and subsequently rinsed three times with PBT for $5 \mathrm{~min}$. Both the $\mathrm{AChE}$ and $\mathrm{TH}$ stained sections were mounted with gelatin on 
SuperFrost Plus slides and allowed to dry overnight. The next day, the slides were dehydrated in an increasing alcohol series (70\%, 95\%, and $100 \%)$ and defatted in xylenes before coverslipping. Sections were inspected, and images were taken with a Leica DM400B digital microscope.

Semiquantitative estimates of combined cholinergic and dopaminergic losses. To determine the extent of cortical cholinergic denervation, an estimate of the number of AChE-positive fibers in cortex was generated (McGaughy et al., 1996; McGaughy and Sarter, 1998; Burk and Sarter, 2001; Burk et al., 2002; Kucinski et al., 2013, 2020). Photographs of AChE-positive fibers in layers $5 / 6$ of primary motor cortex were taken at $40 \times$ magnification. A $4 \times 4$ grid of $30 \mu \mathrm{m}$ (side length) squares was digitally superimposed on each image. Cortical cholinergic fiber density was estimated by manually counting the number of AChE-positive fibers that crossed one of the lines of the superimposed grid using the ImageJ multipoint tool. The extent of cholinergic loss was quantified for each dual lesion animal by subtracting their individual fiber count from the mean fiber count from sham-lesioned rats. This quantification was done for both hemispheres and then averaged to a single value per rat. Based on these data, the degree of AChE-positive fiber loss was scored from 5 to 1 , with 5 indicating the greatest loss of AChE-positive fibers $(5,>90 \%$ loss; $4,>80 \% ; 3,>70 \% ; 2,>60 \% ; 1,30-50 \%$ loss).

For the estimation of striatal TH immunoreactivity (TH-IR) losses, we followed previously described methods (Kucinski et al., 2013, 2015, 2017; Koshy Cherian et al., 2019). Photographs of TH immunostained sections taken at $1.25 \times$ from sections at $\sim 500 \mu \mathrm{m}$ posterior and anterior from 0.2 AP to 1.2 AP, the sites of infusion. TH losses were scored based on the extent and location of the bleaching of TH stains. To assign lesion score based on the extent of TH loss size, TH-IR losses $>2.00 \mathrm{~mm}$ (dorsal-ventral plane $) \times 0.5 \mathrm{~mm}$ (medial-lateral plane) $\times 0.5 \mathrm{~mm}$ (anteriorposterior plane) were assigned the highest score (5). Lower scores $(\leq 4)$ were assigned to smaller lesion sizes (Fig. 1, illustration). We previously determined that $\mathrm{TH}$ losses in the dorsomedial striatum, overlapping with the target field of prefrontal cortical projections are necessary and sufficient to produce falls in DL rats (Kucinski et al., 2013, 2015). Therefore, we also scored the location of the TH losses. TH losses centered closest and mostly restricted to the dorsomedial striatum were assigned the highest score (5), whereas lesions focused more laterally received scores of $\leq 4$ (see Results for illustration). The size and placements scores for both hemispheres were averaged to a single DA lesion score for an individual rat.

Composite (AChE- and TH-loss) depletion score. To generate a score that combines the extent (AChE losses), and extent and location ( $\mathrm{TH}$ losses) of the lesions in DL rats, the two individual scores were multiplied. Therefore, an animal with a complete loss of both cortical AChEpositive fibers, and with large dorsomedial $\mathrm{TH}$ depletions, received a rating of 25 .

\section{Quantification of DREADD transfection space (chromogenic stains)}

Following the completion of behavioral testing, animals were deeply anesthetized using sodium pentobarbital $(270 \mathrm{mg} / \mathrm{kg}$, i.p.) and transcardially perfused with PBS followed by $4 \%$ paraformaldehyde in $0.15 \mathrm{M}$ sodium phosphate buffer with $15 \%$ picric acid, $\mathrm{pH}$ 7.4. Brains were removed and postfixed for $24 \mathrm{~h}$ at $4^{\circ} \mathrm{C}$, and then rinsed in $0.1 \mathrm{M} \mathrm{PBS}$ and stored in $10 \%$ sucrose in PBS solution overnight, followed by $30 \%$ sucrose in PBS solution the following day and allowed to sink. Coronal sections $(40 \mu \mathrm{m})$ were sliced using a freezing microtome (CM 2000R, Leica) and stored in $0.1 \mathrm{M}$ PBS or antifreeze solution until additional processing. Parallel sections were processed for the immunohistochemical visualization or covisualization of mCherry and choline acetyltransferase (ChAT). Immunostaining was performed using primary antibodies (catalog \#632496, Clontech; rabbit anti-DsRed and ab144p, Millipore; goat anti-ChAT and Vectastain Elite ABC kit (catalog \#PK-6100, Vector Laboratories). Sections were first rinsed six times for $5 \mathrm{~min}$ each in PBT, $\mathrm{pH} 7.4$, and then incubated in $1 \%$ peroxide for $30 \mathrm{~min}$. They were rinsed again in PBT three times for $5 \mathrm{~min}$ each before being incubated in the primary antibodies (rabbit anti-mCherry and goat anti-ChAT made in $3 \%$ NDS, 1:5000 and 1:2000, respectively) overnight at $4^{\circ} \mathrm{C}$. The next day, the sections were rinsed three times for $5 \mathrm{~min}$ each in PBT and then incubated in the biotinylated secondary antibody (biotinylated donkey anti-rabbit in $1 \%$ NDS, 1:500) for $60 \mathrm{~min}$. They were then rinsed three times for $5 \mathrm{~min}$ each and then incubated with ABC Elite (1:1000) for $60 \mathrm{~min}$. The sections were again rinsed three times for $5 \mathrm{~min}$ in the PBT before being developed for 10 min using a DAB Peroxidase Substrate Kit [catalog \#SK-4100, Vector Laboratories; made in PBT with $0.024 \% \mathrm{H}_{2} \mathrm{O}_{2}$ and $0.2 \%$ Nickel (II) chloride solution]. The sections were then rinsed three times for 5 min each in PBT and then incubated in the second biotinylated secondary antibody (biotinylated donkey anti-goat in 1\% NDS, 1:500) for $60 \mathrm{~min}$. They were then rinsed three times for $5 \mathrm{~min}$ each and then incubated with ABC Elite (1:1000) for $60 \mathrm{~min}$. The sections were again rinsed three times for $5 \mathrm{~min}$ in the PBT before being developed for 10 min using a DAB Peroxidase Substrate Kit (catalog \#SK-4100, Vector Laboratories; made in PBT with $0.024 \% \mathrm{H}_{2} \mathrm{O}_{2}$ ). Sections were rinsed in PBT three times for 5 min each before being mounted with gelatin onto SuperFrost Plus slides and allowed to dry overnight. The following day, slides were dehydrated in an ascending alcohol series $(70 \%$, $90 \%$, and $100 \%$ ) and defatted in xylene before coverslipping. mCherry- and ChAT-stained slides were imaged using a Leica DM400B digital microscope.

Photographs, at $1.25 \times$ and $5 \times$ magnification, of ChAT-positive and mCherry-positive cells were taken in $400 \mu \mathrm{m}$ increments moving rostrocaudally from AP 1.2 to $0.20 \mathrm{~mm}$. Analogous to the estimation of the extent of cholinergic and DA losses, transfection scores were based on the placement and size of the transfection space. On sections stained for the chromogenic visualization of mCherry and ChAT, single-labeled neurons were stained brown for mCherry and orange for ChAT. Colabeled neurons that expressed mCherry and ChAT appeared black. Circular outlines were superimposed on the area containing double-labeled neurons. Counts taken from these areas were used to generate transfection scores. Transfected areas centered in the dorsomedial striatum were assigned the highest score (5), whereas more lateral placements were assigned scores of $\leq 4$. Transfected areas primarily restricted to the dorsomedial striatum were assigned the highest score (5), whereas areas that spread further were assigned scores $\leq 4$. These scores were then averaged together and plotted against CTTT performance (see Results).

\section{Selectivity of mCherry expression (double immunofluorescence)}

To determine the selectivity of mCherry expression in striatal ChIs, sections were processed for the immunohistochemical visualization of mCherry and VAChT. First, sections were rinsed six times for $5 \mathrm{~min}$ each in $0.1 \mathrm{~m}$ PBS, pH 7.3, and then incubated in $0.1 \%$ Triton diluted in PBS for 15 min (Agostinelli et al., 2019). Sections were then rinsed with PBS three times for 4 min each and incubated in 1\% NDS made in PBS for $60 \mathrm{~min}$ at room temperature. The sections were then incubated overnight in the primary antibodies, Invitrogen rabbit anti-mCherry (catalog \#PA534 974, Thermo Fisher Scientific) and goat anti-VAChT (catalog \#abn100; Millipore; 1:500 for both). The following day, sections were rinsed three times for $5 \mathrm{~min}$ in PBS and then incubated in the secondary antibodies, biotinylated donkey anti-rabbit conjugated to Cy3 (catalog \#711-065-152, Jackson ImmunoResearch) and donkey anti-goat conjugated to Alexa Fluor (catalog \#488705-545-147, Jackson ImmunoResearch) for $90 \mathrm{~min}$. Sections were then rinsed three times for $5 \mathrm{~min}$ in PBS and incubated in Cy5 streptavidin for further mCherry amplification (catalog \#SA-1500, Vector Laboratories; $1: 1000$ ). All sections were rinsed three times for $5 \mathrm{~min}$ with PBS, and then were mounted, air dried, and coverslipped with Vectashield Antifade Mounting Medium (catalog \#H-1000, Vector Laboratories). mCherry selectivity for cholinergic cells was assessed using a Zeiss LM 700 Confocal Microscope. Images taken from the confocal microscope were used to determine the specificity of DREADD expression in ChIs. Images were taken at $5 \times$ magnification from five AP levels $(0.24,0.60,1.08,1.20$, and $1.60 \mathrm{~mm}$ ). Cells in the striatum were counted for mCherry, VAChT, and/or coexpression visualized through mCherry amplification (red), and Alexa Fluor 488 (green) fluorescence. Cells that coexpressed mCherry and VAChT fluoresced a light orange-yellow color due to the simultaneous excitation of both red (mCherry) and green (VAChT) fluorophores. To express the selectivity of DREADD expression in ChIs, within the expression region, as determined based on the chromogenic stains, the number of double- 

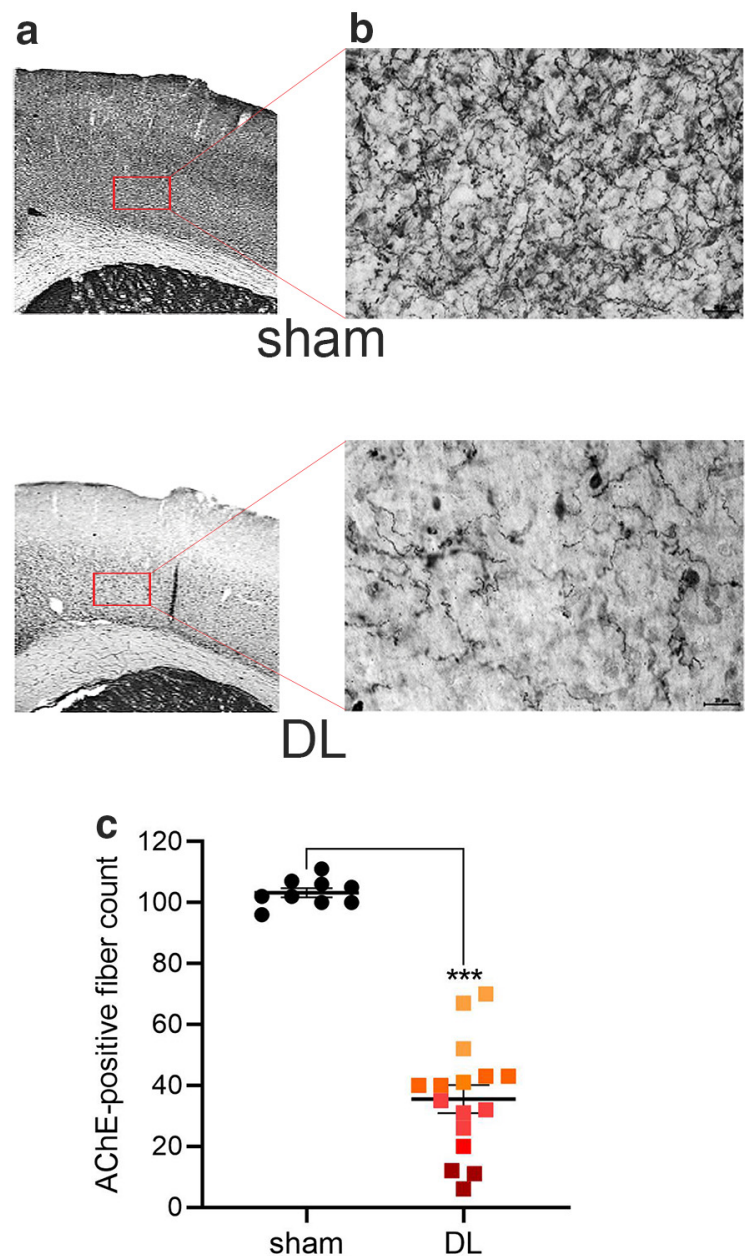

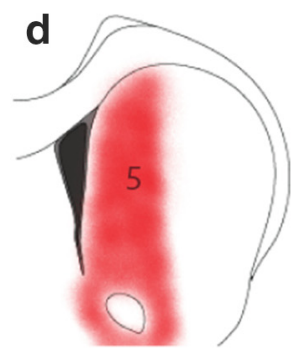

g

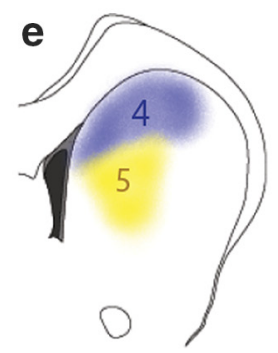

h

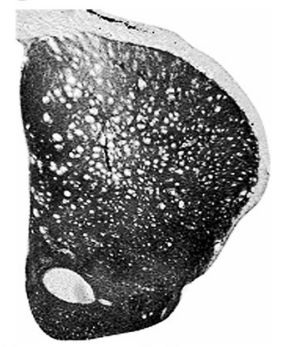

Bregma: $0.2 \mathrm{~mm}$

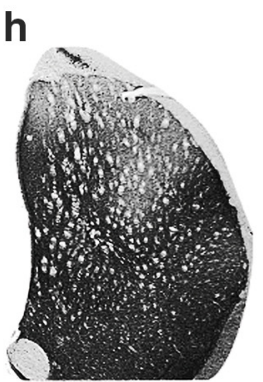

$1.2 \mathrm{~mm}$

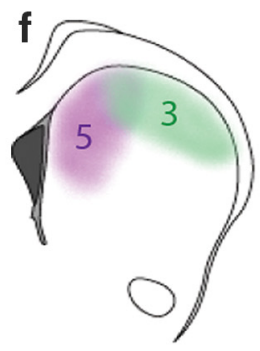

i

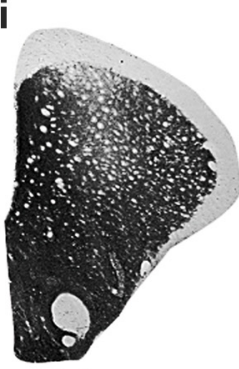

$1.6 \mathrm{~mm}$
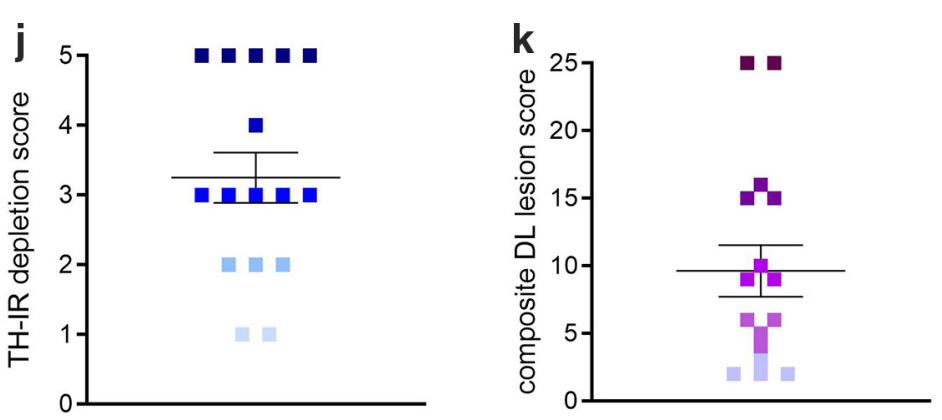

Figure 1. Combined cortical cholinergic and striatal dopaminergic deafferentation (DL rats). $\boldsymbol{a}$, Representative images of coronal sections from a sham-lesioned (top) and a DL rat (bottom), exemplifying the normal density of AChE-positive fibers in the cortex (top) and following deafferentation (bottom; (magnification: $5 \times$ ). The inserts in $\boldsymbol{a}$ are magnified in $\boldsymbol{b}$ (40 $\times$; $25 \mu$ m-scales inserted). Residual AChE-positive fibers were counted using a grid method and losses were scored (see Materials and Methods). c, The color-coded symbols signify the extent of AChE-positive fiber losses, with scores of 1 , orange, denoting a $\geq 50 \%$ loss, to scores of 5 , dark red, denoting $\geq 90 \%$ loss of fibers compared with counts from sham-lesioned rats. $\boldsymbol{d}$, Illustration of the prelimbic projection field in the dorsomedial striatum (Mailly et al., 2013; Hart et al., 2018b), which was the primary target of dopamine depletions in DL rats. Depletion of this region was previously demonstrated to be sufficient for causing falls in DL rats (Kucinski et al., 2013, 2015). Depletion of TH-IR was scored based on placement, with high scores for depletions in the target region, and for the extent of the depletions (see Materials and Methods). Thus, an ideal depletion as shown in $\boldsymbol{d}$ would receive the highest score (5). $\boldsymbol{e}, \boldsymbol{f}$, Illustration of the scoring of striatal TH-IR losses based on the extent and location of losses on sections from bregma $0.2-1.6 \mathrm{~mm}$. The colors designate placement ratings with yellow and pink lesions rated 5, whereas blue and green lesions were only partially localized within the target area. $\boldsymbol{g}$-i, Examples of TH-IR depletions, as visualized on TH-immunostained sections, scored 5 for placement and size (g), 3 and 5 (h), and 4 and $3(\boldsymbol{i})$. $\boldsymbol{j}$, Individual striatal TH-IR loss scores plotted and color coded, with the lowest score (1) in light color and the highest score (5) in dark blue. $\boldsymbol{k}$, Composite cholinergic and dopaminergic lesion scores (see Materials and Methods for calculating the composite score) plotted with lower to higher scores and color coded light to dark purple. As shown in Figure 2, $e$ and $f$, composite scores and TH-IR losses, but not AChE fiber counts, were correlated with residual turn rates (see Results for main statistical findings; pairwise comparisons in this and other figures: $\left.{ }^{*} p<0.05,{ }^{* *} p<0.01,{ }^{* * *} p<0.001\right)$.

labeled neurons (mCherry-positive + VAChT-positive) was divided over the total number of mCherry-expressing neurons (double-labeled + mCherry-positive only).

Expression of $m$ Cherry in wild-type Long-Evans rats following striatal infusions of AAV-hSyn-DIO-hM4D(Gi)-mCherry. Based on chromogenic stains, we observed dark-stained, and thus presumably construct-expressing, neurons in the area around the tip of the infusion needle. Because of the relatively close proximity of these cells to each other, the relatively small diameter of their somata when compared with ChIs (see Fig. 5e), and the absence of such cells on immunostained sections, we also conducted histologic analyses on sections of non-ChAT-Cre-expressing control rats (WT rats). These rats also received infusions of the DREADD construct, using the methods described above.

Overlap between TH-IR depletion and transfection spaces. As we observed greater efficacy of the excitatory hM3Dq DREADD in DL rats with TH-IR losses not overlapping with the transfection space, the overlap between the area of the transfection space and the area of TH-IR loss was determined using the Threshold and Measure tools in ImageJ (version 1.48). The Threshold tool was used to differentiate the two regions, and the surface area of these spaces was measured using the ImageJ Measure tool. Surface area measures were taken on sections from three AP levels $(0.24,1.08$, and $1.20 \mathrm{~mm})$ and averaged. Pixel resolution was converted to micrometers $(1919 \times 1199$ to $2500 \times 1562 \mu \mathrm{m})$ based on a micrometer scale inserted using LAS X microscope imaging software (version 3.41; Leica Microsystems). The overlap between transfection space and TH-IR-depleted areas was calculated by dividing the surface area of the area of overlap by the total surface area of the TH-IR loss.

\section{Experimental design and statistical analysis}

CTTT performance measures (accuracy, latency, and distribution) were compared between the sham and dual lesion groups using within-subjects repeated-measures ANOVAs. Session day was treated as a repeated-measures within-subjects factor. Turn cue modality (auditory or visual) and group were analyzed as between-subjects factors. CTTT performance measures (accuracy, latency, and distribution) were 
compared between the inhibitory and control DREADD groups using within-subjects repeated-measures ANOVAs. CNO (or vehicle) was used as a repeated-measures within-subjects factor. Turn cue modality (auditory or visual) was used as a within-subjects factor. MCMCT performance measures (falls, traversal time, and slips) were compared between rats expressing the inhibitory, or excitatory, and control DREADD virus using within-subjects repeated-measures ANOVAs. CNO (or vehicle) was used as a repeated-measures within-subjects factor. Direction of rotation ( $\mathrm{cc}$ or $\mathrm{cw}$ ) was used as a within-subjects factor when applicable. For the analysis of the beneficial efficacy of the excitatory DREADD, lesion status (DL vs sham) was analyzed as an additional between-subjects factor. Significant turning preferences in the open field were determined using the binomial test $(p=0.50)$, and the number of animals per turning category (preferred left, right, or no significant preference) was compared among the four experimental conditions using the $\chi^{2}$ test. An additional analysis of the turning behavior of the rats, regardless of significantly preferred directions, was conducted using an ANOVA on the effects of group (hM4Di/mCherry) and drug (vehicle/ $\mathrm{CNO}$ ). Assumptions underlying the statistical model were assessed. In cases of the violation of the sphericity assumption, Huyhn-Feldt-corrected $F$ values, along with uncorrected degrees of freedom, are given. Post hoc analyses for all within-subject comparisons and additional analyses were performed using the $t$ test or least significant difference test. Additional statistical tests are justified in Results. Statistical analyses were performed using SPSS for Windows (version 17.0; SPSS). Alpha was set at 0.05 . Exact $p$ values are reported as recommended previously (Greenwald et al., 1996; Sarter and Fritschy, 2008). Effect sizes (Cohen's d) were computed for major results (Cohen, 1988).

\section{Results}

\section{Cued turning, but not stopping, is disrupted in DL rats}

Rats with dual striatal DA and basal forebrain cholinergic losses (DL rats) were previously demonstrated to exhibit elevated fall rates on the MCMCT. Losses of only cholinergic or DA neurons did not cause falls, and fall rates were correlated with impairments in attentional performance. Falls in DL rats were concluded to reflect disruption of normally compensatory attentional supervision of gait and complex movement in rats with impaired striatal function (Kucinski et al., 2013, 2017; Sarter et al., 2014; Koshy Cherian et al., 2019). The first of the present experiments was designed to expand the validity of the conclusions derived from prior experiments using the MCMCT, by testing the hypothesis that cued turning is disrupted in DL rats. Similar to the attention-demanding traversal of a rotating rod (MCMCT), cued turning requires the detection and integration of exteroceptive and proprioceptive cues to guide the selection and sequencing of complex movements (Pavcic et al., 2014; Oh et al., 2018). Turning is impaired in Parkinsonian fallers (Stack and Ashburn, 2008; Cheng et al., 2014; Gilat et al., 2015; Stuart et al., 2017; Virmani et al., 2018).

Cortical cholinergic and striatal TH-IR losses in DL rats were quantified as described in Materials and Methods and previously (Kucinski et al., 2013, 2017; Koshy Cherian et al., 2019; Fig. 1). The density of cholinergic fibers in the cortex of DL rats on average was reduced by $65 \%\left(t_{(18)}=14.05, p<0.0001\right.$; Fig. $\left.1 a-c\right)$. Striatal DA depletions, indicated by TH-IR losses, were scored based on placement and size, as described in Materials and Methods (Fig. $1 d-j$ ). Finally, cortical cholinergic and striatal DA losses were combined into a composite DL lesion score (Fig. 1ks; for details, see Materials and Methods).

Rats were trained to criterion to use the turn and stop cues before surgery (Table 1, Fig. $2 a$ ). Cued turns during the cue-totreadmill stop period did not differ between rats assigned to subsequent sham versus DL surgery $\left(t_{(23)}=0.52, p=0.61\right.$; turns/turn cue trials: sham assigned, $0.83 \pm 0.04$; DL assigned, $0.80 \pm 0.04$ ).
Data from the last four sessions of postsurgery testing (Table 1) were used for the analysis of turning performance in DL rats. Compared with sham-lesioned rats, DL rats failed to turn in response to the turn cue significantly more frequently than shamoperated controls [main effect of group: $F_{(1,21)}=95.57, p<0.01$; ratio of cued turns to trials (mean \pm SEM): shams, $0.77 \pm 0.02$; DL, $0.36 \pm 0.07$; Cohen's $d=5.18$; Fig. $2 b$ (note that Fig. $2 b$ does not illustrate this main effect of group but depicts the data from all four test sessions to indicate the reliability of the group difference)]. There were no effects of cue modality (tone or light), test day, or sex, and no significant interactions among these factors (all $F<1.83$, all $p>0.13$ ). In contrast to cued turns, DL rats stopped in response to stop cues as reliably as sham-lesioned rats (main effect of group: $F_{(1,21)}=0.97, p=0.42$; interactions with cue modality, sex, day: all $F<1.34$, all $p>0.40$; Fig. $2 c$ ).

A secondary analysis discerned the degree to which turns and stops were instigated immediately by the cue or occurred later in response to the treadmill stop and during the pause. As there were no effects of test day in the primary analysis (above), responses were averaged per rat across all four final testing days and expressed as ratios (turns or stops over total turns or stops, respectively) per task phase. Approximately $80 \%$ of cued turns by sham-operated rats occurred in response to the cue during the cue onset-to-treadmill stop period. In contrast, a relatively low number of residual turns in DL rats took place during this period ( $\sim 10 \%$ of residual turns; $t_{(23)}=16.08$; $p<0.01$; Cohen's $\mathrm{d}=7.10$; Fig. $2 d$ ). Residual turns by DL rats during the cue onsetto-treadmill stop period also tended to occur later in that period when compared with sham-operated rats (time from cue onset to turn onset; shams, $0.99 \pm 0.36 \mathrm{~s} ; \mathrm{DL}, 1.29 \pm 0.33 \mathrm{~s} ; t_{(18)}=1.96$, $p=0.07$; the lower degrees of freedom for this comparison reflects the fact that five DL rats did not generate any turns during this period). In response to stop cues, both groups of rats rarely stopped before the treadmill stop (proportion of stops during the cue-to-treadmill stop period over total stops; $t_{(23)}=1.16$; $p=0.22$; Fig. $2 d$ ), indicating that rats used the actual treadmill stop, rather than the stop cue, to cease forward movement.

\section{Correlations between performance measures and cholinergic and DA losses}

Relatively larger composite, cholinergic and TH-IR losses were significantly correlated with lower turn rates $\left(R^{2}=0.26\right.$; $p=0.041$; Fig. 2e). Moreover, individual DA lesion scores $\left(R^{2}=\right.$ $0.48 ; p=0.0029$; Fig. $2 f)$, but not cholinergic lesion scores $\left(R^{2}=\right.$ $0.04 ; p=0.44$; Fig. $2 g$ ), were significantly correlated with turn rates. This finding mirrors the results of an earlier analysis of the relative contributions of cholinergic and DA losses to falls (Kucinski et al., 2013), suggesting that removal of basal forebrain-cortical cholinergic projections unmasks the impact of striatal DA losses.

In summary, the evidence from this experiment indicated that DL rats, which were previously demonstrated to exhibit relatively high fall rates on the MCMCT, also exhibit a robust deficit in cued turning. This finding expands the usefulness of DL rats as a model of the disruption of the cognitive-motor interface in PD fallers, and it also demonstrates the usefulness of the CTTT to model the turning deficits present in Parkinsonian fallers (references above). As the CTTT can be shielded, this task significantly assists in advancing research on PD falls to neurophysiological and neurochemical recording studies. Furthermore, as will be demonstrated below, converging evidence from both tasks indicates an essential role of striatal ChIs in the integration of cognitive-motor functions. 

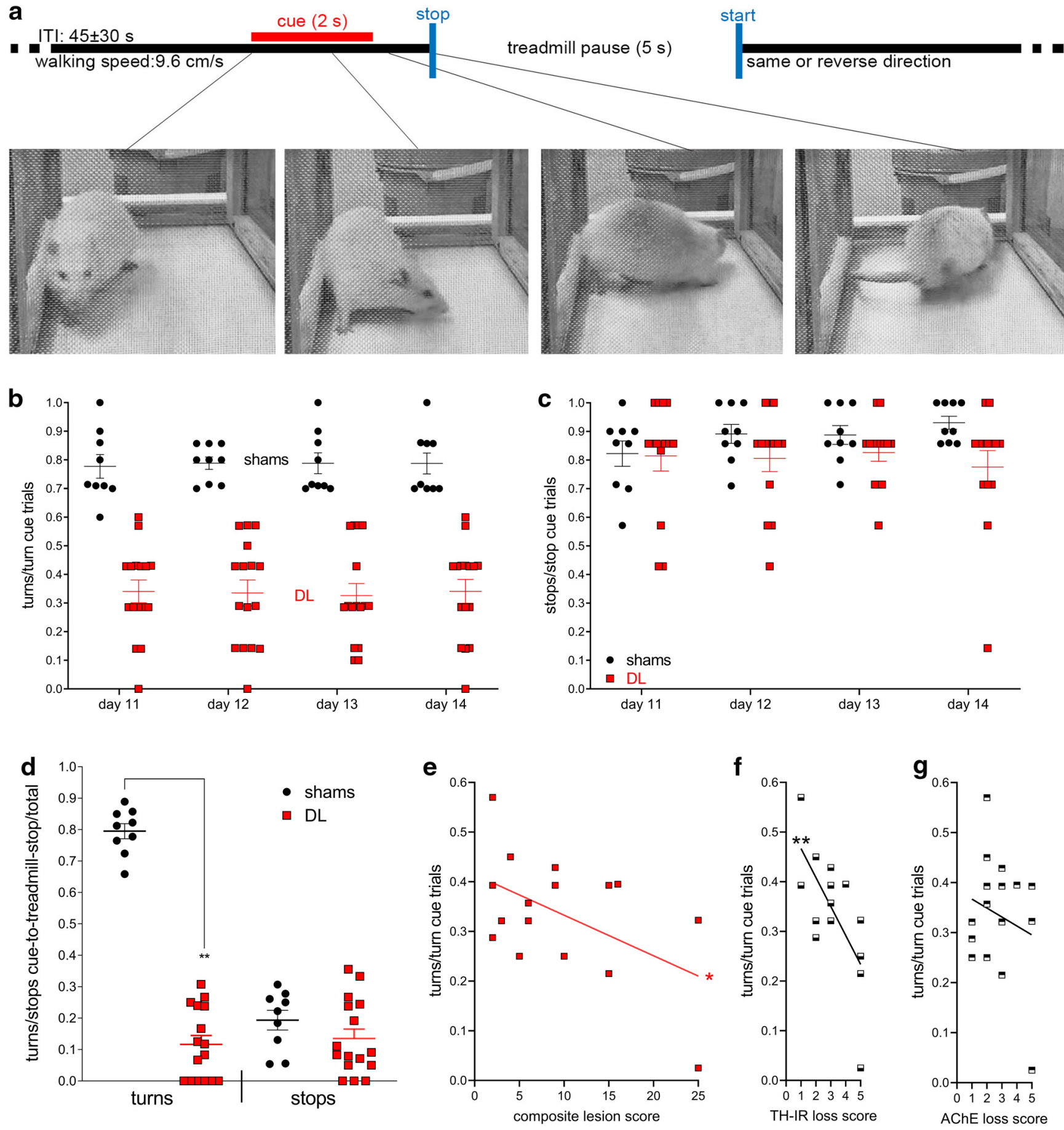

Figure 2. Illustration of the CTT and results indicating impaired cued turning by DL rats. $\boldsymbol{a}$, The CTTT required rats to walk on a treadmill (walking speed, $9.6 \mathrm{~cm} / \mathrm{s}$ ) and to use one cue (tone or light) as a turn cue, indicating that the treadmill will stop and restart, $5 \mathrm{~s}$ later, in reverse direction. The other cue (light or tone) predicted a treadmill restart in the same direction (no rewards were delivered). The photographs show a rat initiating the turn within $500 \mathrm{~ms}$ of the cue onset (see lines connecting the photographs to the task timeline) and completing this turn by the time the treadmill stopped. Cues were separated by a variable ITI of $45 \pm 30 \mathrm{~s}$, during which the rat continued walking the treadmill. Rats were trained to criterion performance before surgery (Table 1). $\boldsymbol{b}$, Cued turns by sham rats $(n=9)$ and DL rats $(n=16)$ during the last $4 \mathrm{~d}$ of postsurgery (TTT practice [all data graphs show individual data and, where appropriate, means (lines) and SEMs (error bars)]. Upon a turn cue, DL rats reliably failed more often than sham rats to produce a successful turn before the treadmill restart (note that $\boldsymbol{b}$ does not illustrate this main effect of group but depicts the data from all four test sessions to indicate the reliability of the group difference, as was indicated by the absence of an interaction between the effects of group and test day). $\boldsymbol{c}$, DL rats stopped on the presentation of a stop cue as reliably as sham controls. $\boldsymbol{d}$, Calculated over the total number of successful turns, sham rats executed the overwhelming proportion of turns during the cue onset-to-treadmill stop period, while the residual turns in DL rats rarely occurred during this phase. It was not possible to attribute residual turns in DL rats during the treadmill pause to a specific task event as such turns may have occurred spontaneously while the treadmill paused. In both groups of rats, cued stops rarely occurred before the treadmill stop (d, right half) and thus took place largely during the treadmill pause. $\boldsymbol{e}-\boldsymbol{g}$, Turn rates in DL rats were significantly correlated with the composite lesion score that combines cholinergic and dopaminergic losses $(\boldsymbol{e})$, and also with striatal DA losses alone $(\boldsymbol{f})$, but not with cholinergic losses alone $(\boldsymbol{g}$; note that the data point indicating near zero turns in $\boldsymbol{f}$ was not considered an outlier but removal did not abolish the significance of the correlation). The histologic findings suggested that cholinergic losses unmask the impact of striatal DA losses on performance. 

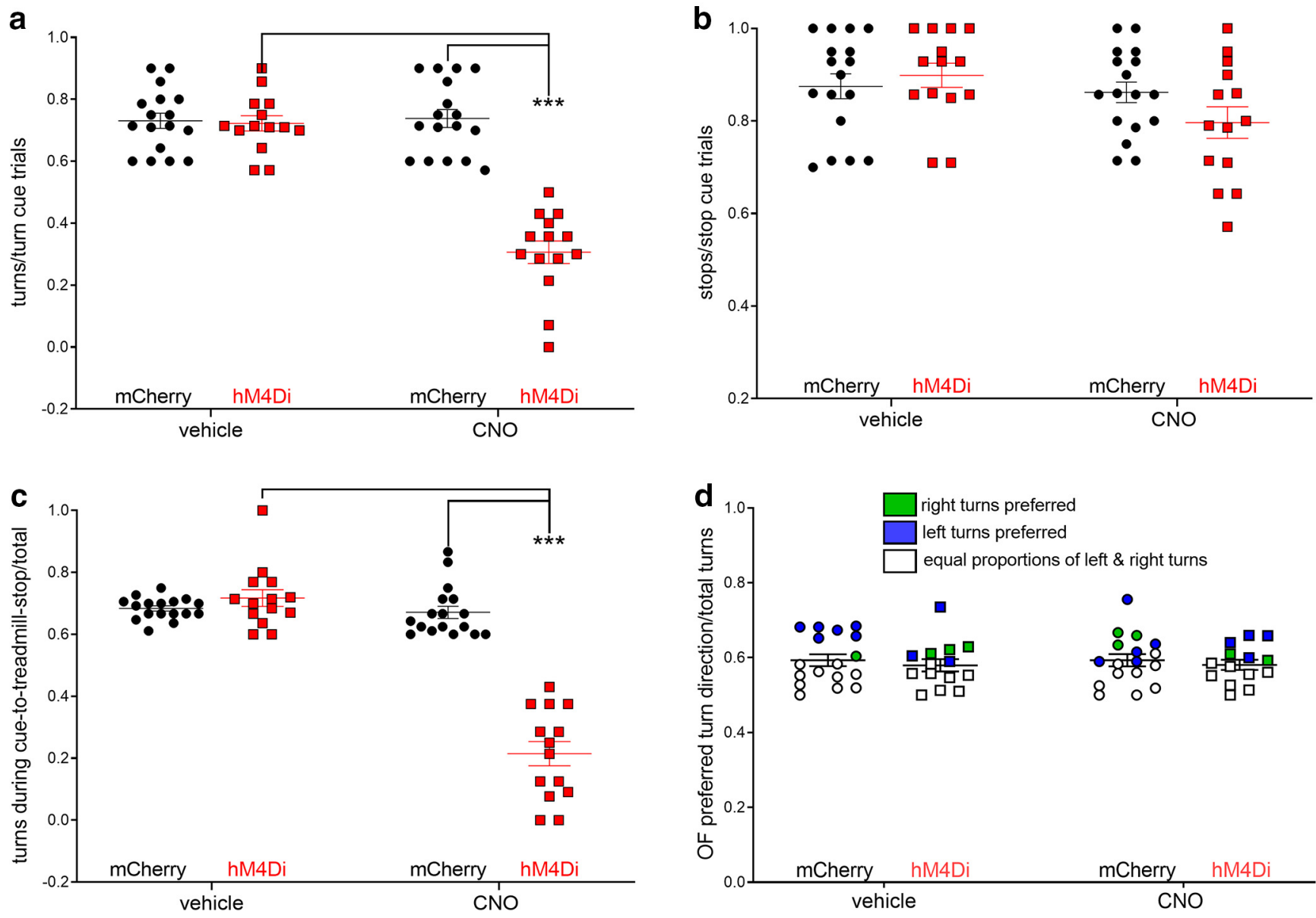

Figure 3. CNO-induced disruption of cued turning behavior in hM4Di-expressing rats performing the (TTT (all graphs show individual values, means, and SEMs). $\boldsymbol{a}$, The proportion of cued turns in trials presenting the turn cue was significantly lower in CNO-treated, hM4Di-expressing rats $(n=14)$ when compared with the effects of CNO in mCherry-expressing control rats $(n=14)$ and the effects of vehicle in hM4Di-expressing rats (for ANOVAs, see Results). $\boldsymbol{b}$, In contrast, stops during stop cue-presenting trials were unaffected by CNO or group. $\boldsymbol{c}$, Further paralleling the performance of DL rats in the CTTT (Fig. 2), the majority of the turns in vehicle-treated or mCherry-expressing control rats occurred before the treadmill stop, and CNO given to hM4Di-expressing rats interfered with cue-evoked turns during this task period. $\boldsymbol{d}$, The effects of CNO were also assessed in the rats while exploring an open field, primarily to determine whether the administration of CNO interfered with the preferred turning direction of the rats. The color-coded symbols in $\boldsymbol{d}$ indicate rats with a significant preference (binomial test, $p=0.50$; see Results) for a turning direction; animal numbers per category did not differ between groups and drug. Likewise, an overall analysis of turning preferences, regardless of whether they were significantly different from chance, shown in $\boldsymbol{d}$, did not indicate effects of group or drug, or an interaction between these two factors. Together, these analyses did not support the possibility that the effects of $\mathrm{CNO}$ on turning performance in the $\mathrm{CTTT}(\boldsymbol{a}, \boldsymbol{c})$ were confounded by interfering with their spontaneous and preferred turning behavior.

\section{Cued turning, but not stopping, is disrupted by suppression of striatal ChI activity}

Striatal ChIs are generally thought to participate in the integration of corticostriatal information about task and movement cues with the movement-energizing functions of striatal dopamine signaling (Apicella, 2017; Okada et al., 2018; Gritton et al., 2019). Thus, we hypothesized that suppressing the activity of ChIs in intact rats reproduces, at least partly, the cued turning deficits and the falls seen $\mathrm{DL}$ rats.

The effects of CNO or vehicle were tested in rats expressing the inhibitory hM4Di DREADD construct $(n=14,11$ females) or only mCherry ( $n=17,12$ females), giving rise to a four-factorial mixed-model ANOVA (construct, sex, cue modality, drug). An initial inspection of the data indicated that male and female rats treated with vehicle performed similarly and that CNO produced similar effects on cued turns in male and female rats [turns/cued turn trials (mean $\pm \mathrm{SEM}$ ): vehicle: females, $0.72 \pm 0.03$; males, $0.74 \pm 0.03$; CNO: females, $0.53 \pm 0.04$; males, $0.57 \pm 0.08$ ). Therefore, and to limit the omnibus analysis to a mixed model of the effects of two between-subject (group, modality) and one within-subject factor (vehicle/CNO), the factor sex was removed from the final analysis.
Administration of $\mathrm{CNO}$ to rats expressing the inhibitory DREADD significantly reduced the number of turns in trials that began with the presentation of the turn cue (group $\times$ vehicle/ CNO: $F_{(1,27)}=103.65, p<0.0001$; Cohen's $d=1.96$; Fig. $3 a$ ). Reflecting the large effects of CNO in hM4Di rats, the main effects of group (mCherry/hM4Di) and drug [vehicle/CNO also reached significance (both $F>37.57$, both $p<0.0001$; mean $(\mathrm{SEM})$ ratios of turns/turn trials: mCherry, $0.68 \pm 0.03$; $\mathrm{hM} 4 \mathrm{Di}$, $0.50 \pm 0.03$; vehicle, $0.71 \pm 0.02 ; \mathrm{CNO}, 0.49 \pm 0.05)$. There was no effect of turn cue modality (tone or light) and no interactions involving this factor (all $F<0.42$, all $p>0.52$ ).

As illustrated in Figure $3 b$, cued stop rates did not differ between the rats and treatment conditions (all main effects and all interactions, all $F<4.00$, all $p>0.06$ ).

Paralleling the CTTT performance by DL rats (above), the overwhelming proportion of cued turns of rats expressing the mCherry control construct and given $\mathrm{CNO}$, or of hM4Di rats given vehicle, occurred during the cue onset-to-treadmill stop period. Furthermore, the administration of CNO drastically reduced this portion of cued turns in hM4Di rats (main effects of group, drug and interaction: all $F>51.98$, all $p<0.0001$; Cohen's $d=2.34$ for the effect of CNO in hM4Di vs mCherry 
rats; Fig. 3c). Importantly, in non-DREADD-expressing rats, CNO had no effects on cued turns, cued stops, or open field behavior, which is consistent with our prior evidence indicating the absence of significant off-target effects of this dose of CNO (Kucinski et al., 2019).

\section{Inhibition of ChIs does not interfere with spontaneous turning behavior}

In the CTTT, rats maintained a preferred turning direction (left or right, from the perspective of the rats) to execute successful turns. Therefore, we were concerned about the possibility that inhibition of striatal ChIs neurons interfered with the execution of turns in the preferred direction of the rat. Such an effect could have confounded the effects of suppression of ChI activity on cued turning in the CTTT. We determined the spontaneous turning preferences of the rats during exploration of an open field and following the administration of vehicle, and tested the hypothesis that the administration of $\mathrm{CNO}$ to hM4Di-expressing rats significantly affects the proportion of preferred turns. During the 3 min exploration period of the open field, rats exhibited a total of $51.00 \pm 3.17$ turns (no effects of group and drug and no interaction; all $F_{(1,29)}<1.71$, all $p>0.20$ ). We then determined the number of rats per group that exhibited a preferred turning direction (left or right; binomial test, with $p=0.50$ ). The majority of rats in each group did not exhibit a significant turning preference, and the distribution of rats per category did not differ significantly between the groups (number of rats per group preferably turning right, left, or exhibiting no preference: vehicle/mCherry: 2, 6, 9; vehicle/hM4Di: 3, 3, 8; CNO/mCherry: 5, 3, 9; CNO/hM4Di: 4, 2, 8; $\left.\chi^{2}=3.34(6, N=60), p=0.76\right)$. Because of the relatively low number of rats per category, we conducted a secondary analysis of the effects of group and drug on the proportion of preferred turns, regardless of whether such a preference was significant or not (Fig. $3 d)$. Individually, all rats of all groups exhibited a mild preference ( $\sim 60 \%$ of turns) for a particular turning direction or for no particular direction (Fig. $3 d$ ). Neither group nor drug differentiated this measure of preference (main effects and interaction: $F_{(1,29)}<1.71$, all $p>0.20)$. Furthermore, analyses of secondary measures of the open field behavior of the rats indicated the absence of effects of group and drug, and of interactions between these two factors, on the total distance traveled during the $3 \mathrm{~min}$ exposure to the open field (all $F_{(1,29)}<0.97$, all $p>0.33$ ), locomotor speed (all $F_{(1,29)}<$ 1.09 , all $p>0.31$ ), and the relative time spent in the central quadrant of the field (all $F_{(1,29)}<3.07$, all $p>0.09$ ). Together, these data did not indicate the effects of activating the hM4Di construct in striatal ChIs, or of the effects of $\mathrm{CNO}$, on the spontaneous turning and exploratory behavior of the rats.

\section{Inhibition of striatal ChIs causes falls on the MCMCT}

Following presurgery MCMCT practice (Table 2; Fig. 4a, illustration of the MCMCT), rats received bilateral infusions of the DREADD or the control construct into the dorsomedial striatum. Rats then underwent $5 \mathrm{~d}$ of testing on the traversal sequence described in Table 2. Falls, slips, and traversal time were assessed on the straight rotating rod ( $8 \mathrm{rpm})$ over 4 consecutive test days. First, the rod was rotated in the familiar (counterclockwise) direction (2d), and rats received vehicle injections one day and $\mathrm{CNO}$ the other day according to a counterbalanced design (see Materials and Methods). Rotation direction then was reversed to the unfamiliar (clockwise) direction for $2 \mathrm{~d}$ and rats again received vehicle one day and $\mathrm{CNO}$ the other day.

Following the administration of $\mathrm{CNO}$, fall rates were generally higher than those following vehicle administration (main effect of CNO: $F_{(1,23)}=5.78, p=0.03$; vehicle $0.61 \pm 0.06$ falls/ run; $\mathrm{CNO}, 0.87+0.09$ falls/run; Cohen's $\mathrm{d}=0.63)$. Importantly, a significant $\mathrm{CNO} \times$ group interaction $\left(F_{(1,23)}=5.12, p=0.03\right)$ reflected the disruptive impact of $\mathrm{CNO}$ in rats expressing the inhibitory hM4Di DREADD $\left(F_{(1,11)}=11.54, p=0.006\right.$; Cohen's $\mathrm{d}=1.39$ ) and the lack of effects of $\mathrm{CNO}$ in rats expressing the control construct $\left(F_{(1,11)}=0.01, p=0.92\right.$; Fig. $\left.4 b\right)$. The main effects of group and rotation direction were not significant (both $F<1.05$, both $p>0.31$ ), and there were no two-way or threeway interactions among group, $\mathrm{CNO}$, and rotation direction (all $F<1.71$, all $p>0.20$ for all).

In the presence of $\mathrm{CNO}$, the number of slips also increased significantly (main effect of CNO: $F_{(1,23)}=6.21, p=0.02$; vehicle, $2.65 \pm 0.28 \mathrm{slips} / \mathrm{run}$; CNO, $4.07 \pm 0.45 \mathrm{slips} /$ run $)$. However, the $\mathrm{CNO} \times$ group interaction did not reach significance $\left(F_{(1,23)}=\right.$ 3.11, $p=0.09$; Fig. 4c). As was the case for falls, there were no effects of group and rotation direction, and no other interactions among the factors (all $F<2.78$, all $p>0.09$ ).

Traversal time, which was corrected by the time consumed for falls (see Materials and Methods), was not significantly affected by CNO (main effect of CNO: $F_{(1,23)}=3.96, p=0.06$; vehicle:, $7.84 \pm 0.84 \mathrm{~s}$; $\mathrm{CNO}, 9.45 \pm 0.76 \mathrm{~s} ; \mathrm{CNO} \times$ group: $F_{(1,23)}=$ $1.30, p=0.27$; Fig. $4 d$ ). Unexpectedly, rats expressing the inhibitory DREADD construct were significantly slower than control construct-expressing rats (main effect of group; $F_{(1,23)}=6.21$, $p=0.02$; mCherry, $7.42 \pm 0.73 \mathrm{~s}$; hM4Di, $9.88 \pm 0.65 \mathrm{~s}$ ), but this effect did not interact with any other factor, and there were no other interactions (all $F<1.72$, all $p>0.20$ ).

Given the range of fall rates across hM4Di-expressing rats given $\mathrm{CNO}$, we assessed, post hoc, the possibility that CNO-induced increases in fall rates occurred primarily in rats with relatively poor traversal performance at baseline (following the administration of vehicle). However, these two sets of data were not correlated $\left(R^{2}=\right.$ $0.001, p=0.98)$. Furthermore, and consistent with the prior observation that slips and relatively slow traversal time were correlated with fall rate (Kucinski et al., 2013), correlations between falls and slips, and between falls and traversal time, were significant for both hM4Di-expressing and mCherry-expressing rats given $\mathrm{CNO}$ (mCherry: both $R^{2}>0.48$, both $p<0.01$; hM4Di: both $R^{2}>0.58$, both $p<0.05$ ). These correlational analyses indicate that the efficacy of CNO in hM4Di-expressing rats was unrelated to the baseline (vehicle) performance of the rats, and that relatively higher slip rates and slower traversal time may have contributed to the increase in falls seen after CNO. Additional inspection of individual MCMCT runs of hM4Di-expressing rats exhibiting particularly high fall rates after $\mathrm{CNO}$ did not reveal a specific behavioral effect of $\mathrm{CNO}$, which could be associated with, or even considered a causal variable for, falls. These falls are interpreted as reflecting disrupted striatal integration of movement cues, movement selections and sequences (see Discussion).

\section{Striatal expression of hM4Di}

DREADD transfection regions were visualized and scored based on chromogenic stains (Fig. 5), and the number of mCherry-, VAChT- and double-labeled neurons were counted in these regions (Fig. 6). Higher transfection scores, depicting larger transfection areas that were located within the dorsomedial striatum (for scoring procedures, see Materials and Methods; Fig. $5 a-c$, illustrations) were significantly correlated with greater cued turning deficits following the administration of CNO (Fig. $5 f$ ). As illustrated in Figure 5, $d$ and $e$, dark-stained cells, and thus presumably double-labeled cells, which, however, were relatively closely spaced and of smaller, roundish diameter compared 
a

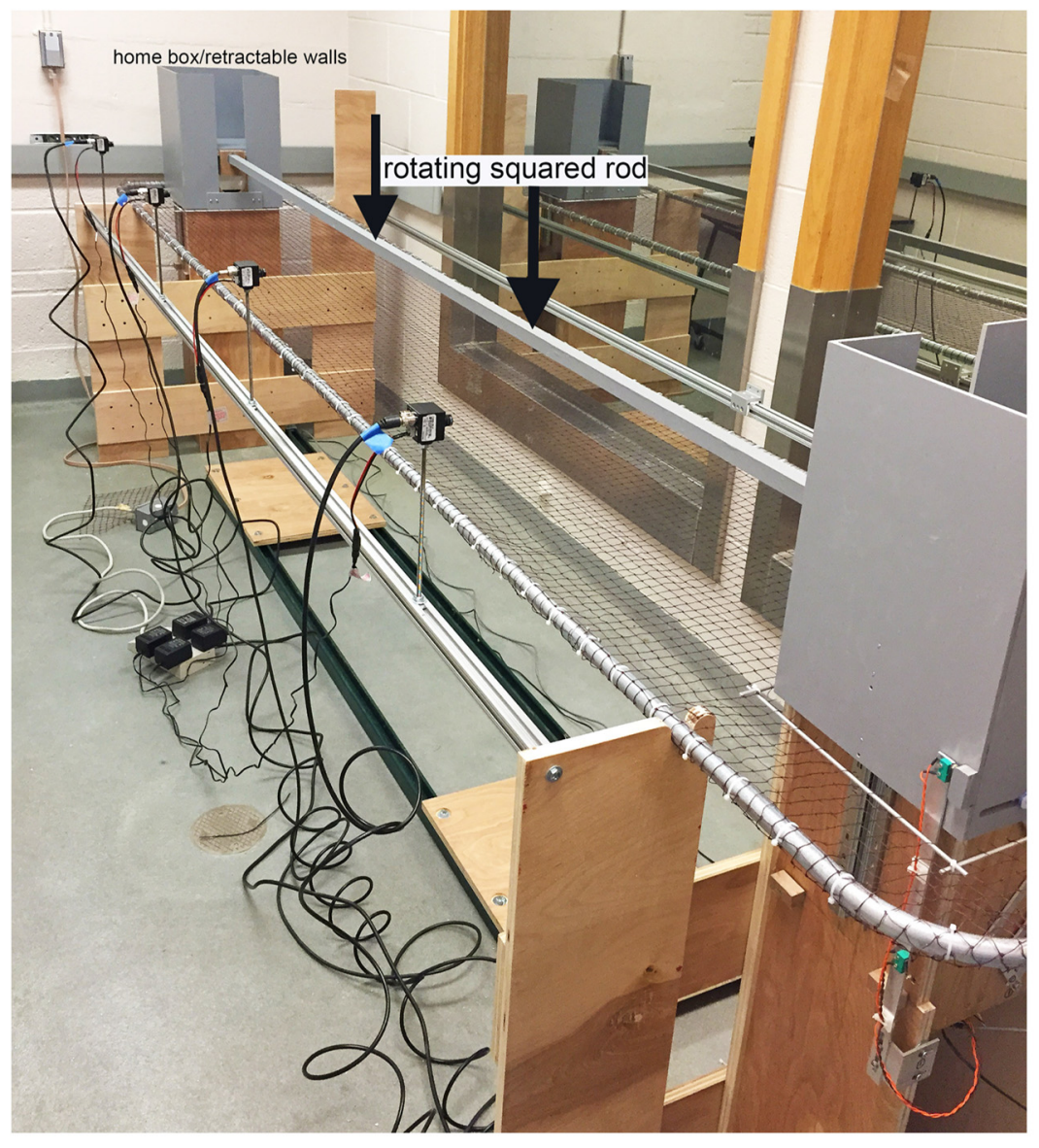

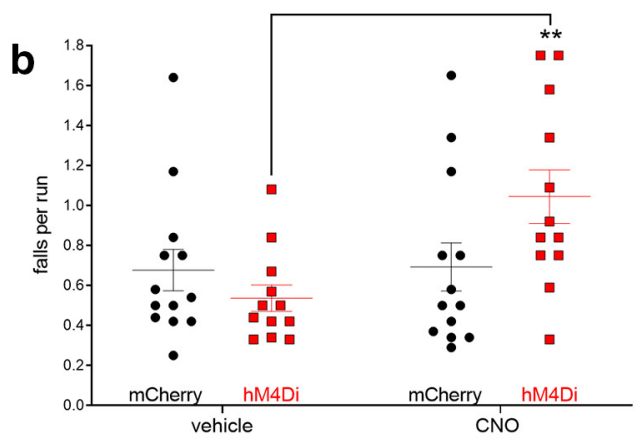
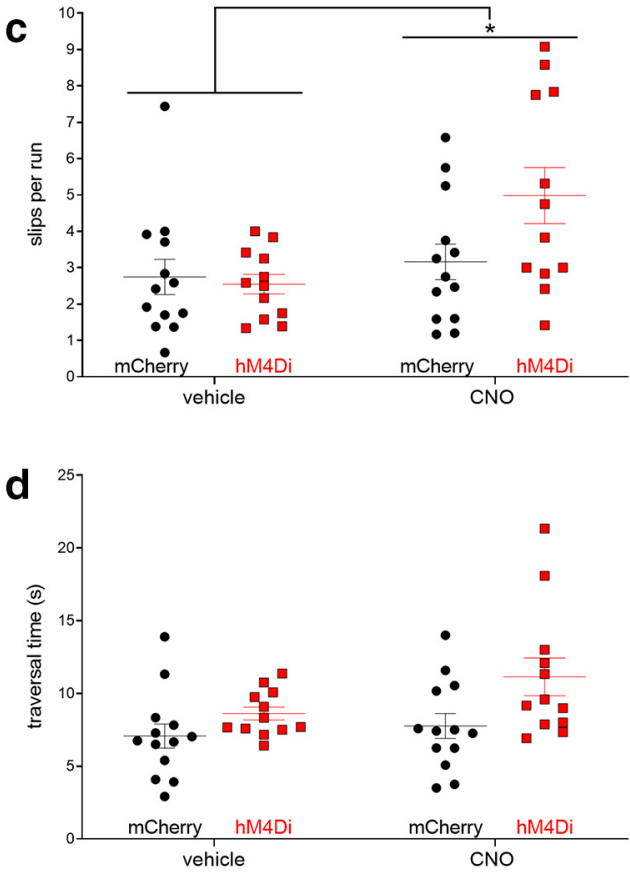

Figure 4. a, Illustration of the 3-m-long MCMCT, with the rotating squared rod installed, and the two home boxes, with their retractable walls shown on both ends of the rod. The three cameras used for recording the runs can be seen on the left along the extension of the rod. Actual falls—into the net—were prevented by the experimenter whenever possible to promote completion of the run (but counted as falls; for details, see Materials and Methods). $\boldsymbol{b}$, In rats expressing the inhibitory hM4Di $(n=12)$, administration of CNO resulted in higher fall rates than in rats expressing the mCherry-control construct ( $n=13$; all graphs show individual data, means, and SEMs; see Results for ANOVAs and effect sizes). c, CNO also produced more slips, but the interaction between virus construct type and CNO/vehicle failed to reach significance. $\boldsymbol{d}$, There were no effects of CNO on traversal time.

with ChIs, were observed within the vicinity of the infusion needle tip (Fig. $5 e$, gray arrows). Such label was also observed on sections from WT rats infused with the DREADD construct (data not shown) and thus considered artifactual. Confocal examination and counting of single- and double-labeled neurons (Fig. $6 a-f)$ indicated a high degree $(>90 \%)$ of mCherry expression in VAChT-positive neurons (Fig. $6 g$ ). mCherry-positive immunofluorescence was virtually absent on section from WT rats infused with the active DREADD construct (data not shown).

\section{Rescuing falls and cued turns in DL rats by stimulating ChIs} Given the essential role of ChIs for cued turning performance, as indicated by CTTT performance, and the ability to traverse a highly dynamic surface in the MCMCT, next we hypothesized that the stimulation of ChIs improves the CTTT and MCMCT performance of DL rats. We infused a Cre-dependent excitatory DREADD, hM3Dq, bilaterally into the dorsomedial striatum of rats that, in a separate subsequent surgery, also received infusions of the neurotoxins into the basal forebrain and striatum, to generate DL and sham-lesioned rats with ChIs expressing hM3Dq (Table 3, timeline and sequence of procedures). The MCMCT and CTTT training regimen and the determination of effects of CNO and vehicle are indicated in Tables 1 and 2. Rats first underwent MCMCT training and testing and thereafter CTTT training and testing (Table 3 ).

\section{Striatal expression of $h M 3 D q$ in $D L$ rats}

Similar to the experiment on the effects of activation of an inhibitory DREADD, excitatory DREADD transfection regions were determined and scored based on chromogenic stains (Fig. $7 a-c$ ), and the number of mCherry-, VAChT-, and double-labeled neurons (Fig. $7 d$ ) were counted in these regions. These analyses focused on potential alterations of viral construct expression in striatal regions depleted of DA, and the possibility that certain combinations of DL composite lesion scores and TH-IR scores favored the demonstration of $\mathrm{CNO}$-induced performance improvements. For this latter aspect, we used the effects of CNO on cued turning rates (Fig. $8 c$ ) because of the relatively greater efficacy of $\mathrm{CNO}$ on this measure when compared with effects on falls from the rotating straight rod.

DL composite lesion scores were comparable to scores from the first experiment on CTTT performance by DL rats (Fig. $1 k$; 

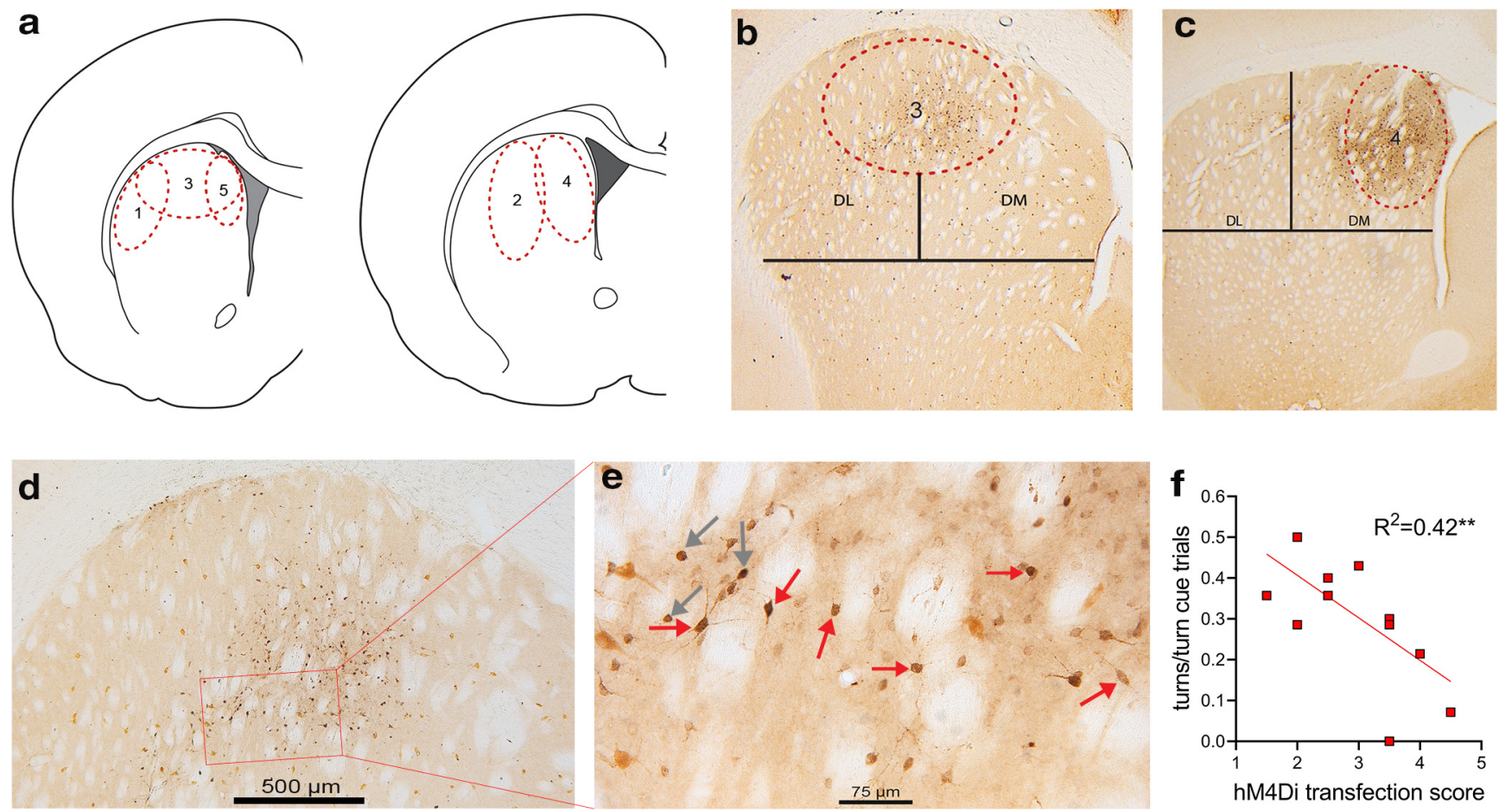

Figure 5. Visualization and scoring of the striatal transfection space (chromogenic stains). $\boldsymbol{a}-\boldsymbol{c}$, Illustrations and examples of the transfection space scoring scheme. On coronal sections, the area exhibiting presumably double-labeled, dark-stained neurons was defined (red, dashed, circular outlines; for details, see Materials and Methods). Transfected areas primarily restricted and localized to the prelimbic projection field of the dorsomedial striatum were assigned the highest score (5), whereas areas that spread further and/or more lateral areas were assigned scores $(\leq 4 ; \boldsymbol{a}, \boldsymbol{b})$. Representative images of coronal sections (magnification, $1.25 \times)$ at AP $1.20(\boldsymbol{b})$ and $0.24(\boldsymbol{c})$ with transfection areas and scores indicated. Lines were superimposed on these images to differentiate and define medial versus lateral, as well as dorsal versus ventral striatum. In $\boldsymbol{b}$, expression was not restricted to the dorsomedial striatum and therefore was assigned a score of 3, whereas in $\boldsymbol{c}$, expression was largely localized in the dorsomedial area but extended laterally and thus was assigned a score of 4 . $\boldsymbol{d}$, Coronal section showing single-labeled (light brown) and double-labeled (dark brown) neurons in the dorsal striatum (score, 3; magnification, 10×). Scale bar, $300 \mu \mathrm{m}$. $\boldsymbol{e}$, Microphotograph of the insert shown in $\boldsymbol{d}$ (magnification, 20×; scale bar, $75 \mu \mathrm{m}$ ), illustrating single-labeled and double-labeled neurons (red arrows exemplify the identification of double-labeled neurons). Within $\sim 100-150 \mu \mathrm{m}$ of the tip of the injection needle, we observed dark brown-labeled, roundish cells (marked gray arrows) that, compared with Chls, appeared to be relatively small in diameter and relatively close together. Such a chromogenic label, close to the needle tip, was also seen on sections from WT rats that were infused with the viral construct (data not shown) and thus considered an artifact. $f$, The sizes of the areas transfected with the inhibitory DREADD construct, indicated by the transfection scores, were significantly correlated with CNO-induced turn rates in the $C T T T\left(R^{2}=0.42 ; p=0.013\right)$.

$\mathrm{U}=67, p=0.52$ ). Likewise, mCherry transfection scores (Fig. $7 c$ ) did not differ significantly from transfection scores from the experiments on the effects of the inhibitory hM4Di DREADD (Fig. $5 k ; \mathrm{U}=118.50, p=0.77$ ). Importantly, mCherry transfection spaces for the excitatory hM3Dq DREADD did not differ between sham-operated and DL rats (colabeled/mCherry labeled neurons: shams, $0.97 \pm 0.004$; DL, $0.96 \pm 0.004$; main effect of group and group $\times$ AP level: both $F_{(1,8)}<4.11$, both $p>0.07$; Fig. 7c). Together, these analyses did not suggest that striatal dopamine depletion altered the expression space and the selectivity of the viral construct by ChIs.

\section{MCMCT}

As detailed in Table 2, rats were familiarized with the cc rotating rod before surgeries but experienced the $\mathrm{cw}$ rotating rod for the first time during the test of effects of $\mathrm{CNO}$ and vehicle. Because of postsurgery complications, one shamlesioned rat was removed from MCMCT testing but included in the subsequent CTTT testing (below). As seen before (Kucinski et al., 2013), traversing the less familiar cw-rotating rod caused more falls in all rats than the cc rotating rod (main effect of direction: $F_{(1,15)}=16.02, p=0.001$; Fig. $8 b$ ). Furthermore, DL rats, treated with the vehicle, fell more frequently than sham rats during traversal of the less familiar $\mathrm{cw}$ rotating rod (Fig. $8 b$, right). Consequently, the fall-reducing effects of CNO in DL rats manifested only in this rotation condition, as reflected by a significant three-way interaction among the effects of lesion status, drug (CNO vs vehicle), and rotation direction $\left(F_{(1,15)}=7.05, p=0.018\right.$; Fig. $\left.8 b\right)$. Follow-up ANOVAs and multiple comparisons on falls during traversal of the $\mathrm{cw}$ rotating rod indicated a significant interaction between the effects of lesion status and drug $\left(F_{(1,15)}=5.42, p=0.034\right)$. This interaction reflected that, following vehicle administration, DL rats fell more frequently than sham rats and, following $\mathrm{CNO}$ administration, this difference was abolished. Moreover, CNOtreated DL rats fell less frequently than vehicle-treated DL rats (Cohen's $\mathrm{d}=1.08$ ), while $\mathrm{CNO}$ had no effect on the fall rate of sham rats (Fig. 8b, illustration of post hoc multiple comparisons; both $p<0.05$; Fig. $7 a$ ).

The three-way interaction found in the analysis of falls was reproduced in the analysis of the number of slips $\left(F_{(1,15)}=11.39\right.$, $p=0.004$; data not shown). Furthermore, the effects of lesion status and drug on slips during $\mathrm{cw}$ rotating rod traversal was significant $\left(F_{(1,15)}=8.23, p=0.012\right)$ and post hoc comparisons indicated more slips in DL than in sham rats $\left(F_{(1,16)}=4.80\right.$, $p=0.045$; shams, $3.09 \pm 0.59$ slips/run; DL, $6.50 \pm 1.22$ slips/ run), but no significant effect of CNO in DL rats on slips $\left(t_{(9)}=\right.$ $3.80, p=0.08$; data not shown). All rats were faster traversing the familiar $\mathrm{cc}$ rotating rod when compared with the $\mathrm{cw}$ rotating rod $\left(F_{(1,15)}=5.00, p=0.041 ; c c, 8.41 \pm 0.75 \mathrm{~s} / \mathrm{run} ; \mathrm{cw}, 10.20 \pm 1.10 \mathrm{~s} /\right.$ run), but there were no effects of, and no interactions between the effects of lesion status, drug, and rotation direction (all $p>0.22$; data not shown). 

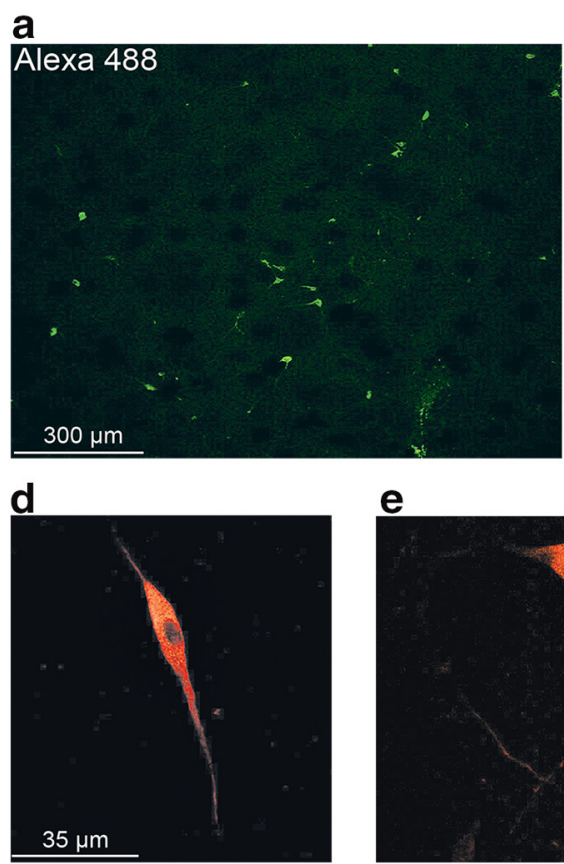

e b
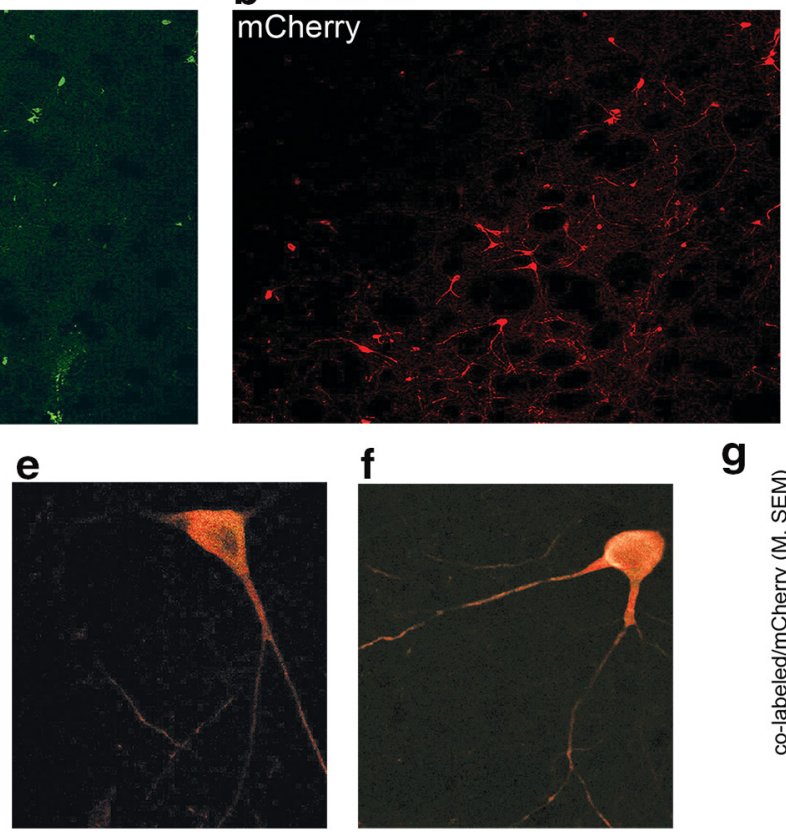

f

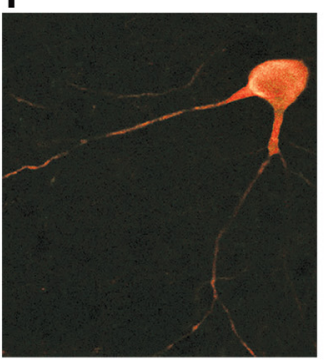

C

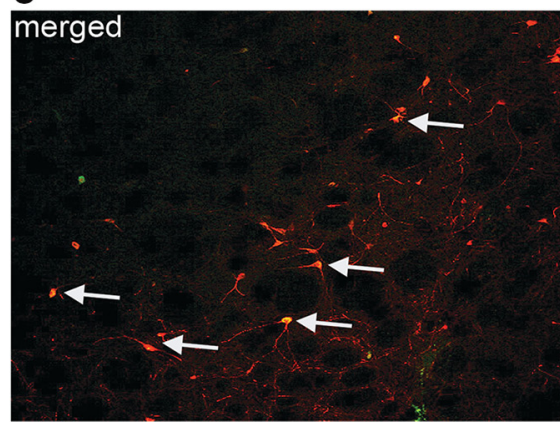

9

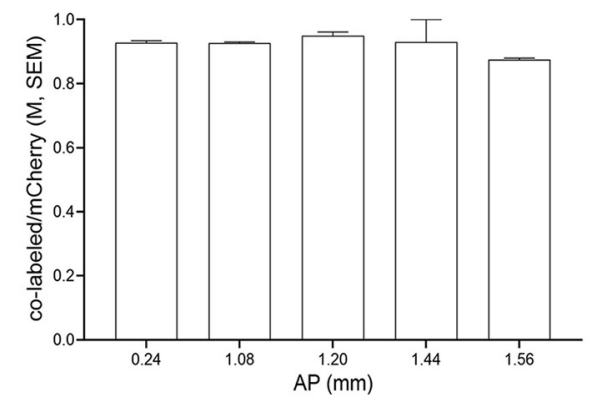

Figure 6. $\boldsymbol{a}-\boldsymbol{c}$, Coronal sections exemplifying VAChT-positive (Alexa Fluor 488), mCherry-positive (DREADD reporter), and double-labeled neurons in the dorsomedial striatum. Scale bar, 300 $\mu \mathrm{m}$. $\boldsymbol{d}-\boldsymbol{f}$, Examples of double-labeled cells. Scale bar, $35 \mu \mathrm{m}$. Single-labeled and double-labeled cells were counted in the transfection regions determined based on chromogenic stains (Fig. 5; see Materials and Methods). g, Over $90 \%$ of mCherry-positive cells were also VAChT-positive (the abscissa depicts the anteroposterior level of sections relative to bregma). Immunostained sections from WT rats infused with the hM3Dq DREADD construct were completely devoid of mCherry-positive cells (data not shown), further supporting the artifactual nature of relatively small, dark-stained cells in the vicinity of the injection needle seen on sections stained for the chromogenic visualization of the transfection space (Fig. 5).

\section{CTTT}

Following a $7 \mathrm{~d}$ pause after completion of MCMCT testing, these rats were also tested on the CTTT. The analysis of the number of cued turns (Fig. 8c) over all trials presenting turn cues (Fig. $8 d$ ), and of cued turns occurring in the period from the cue to the treadmill stop (Fig. 8e), indicated significant interactions between the effects of drug ( $\mathrm{CNO}$ vs vehicle) and lesion status (shams vs DL; both $F_{(1,16)}>36.56$, both $p<0.0001$, both $\mathrm{d}>1.94$; main effects of drug and lesion status: all $F>36.56$, all $p<0.001)$. In both cases, post hoc multiple comparisons indicated significantly lower rates of cued turns in DL rats than in sham rats (both $t_{(16)}>3.50$, both $p<0.0001$ ), and significantly more cued turns in DL rats treated with CNO compared with vehicle (both $t_{(16)}>7.02$, both $p<0.0001$, both $\mathrm{d}>1.44$ ). Cued stops did not differ by lesion status, and $\mathrm{CNO}$ had no effects on this measure (all $F_{(1,16)}<1.39$, all $p>0.26$; Fig. $8 f$ ).

In $\mathrm{DL}$ rats, the $\mathrm{CNO}$-induced reductions in falls on the $\mathrm{cw}$ rotating straight road, relative to the performance of these rats following vehicle administration, did not significantly correlate with the CNO-induced increases in cued turns (either total or when restricted to the cue-to-treadmill stop period; all $p>0.69$ ). Inspection of the data from the two tasks (Fig. 8) indicated that individual turn rate data from DL and sham rats did not overlap or only partly overlapped, while fall rates from the two groups overlapped considerably. Thus, the CTTT appeared to reveal the impairments in DL rats with relatively greater sensitivity and efficacy, and this may have limited the manifestation of correlations between performance measures from the two tasks.

\section{Test of off-target effects of CNO in DL rats}

To assess potential off-target effects of $\mathrm{CNO}$, a separate group of DL rats ( $n=8$ females), expressing the mCherry-positive, nonDREADD control construct, was prepared. These rats first were tested over 8 runs each on the $\mathrm{cc}$ and $\mathrm{cw}$ rotating rods of the
MCMCT ( $8 \mathrm{rpm} ; 16$ runs total). Vehicle or CNO was administered on alternating test days so that vehicle was administered before half of the runs and CNO before the other half (paralleling the procedures listed in Table 2). The administration of CNO did not affect traversal time, slips, or fall rates (all $t_{(7)}<0.80$, all $p>0.40)$. These animals were then also trained and tested on the CTTT, similar to the procedures shown in Table 1 (right). The administration of CNO to non-DREADD-expressing DL rats affected neither their cued turning nor stop rates (both $t_{(7)}<$ 2.33 , both $p>0.17$; data not shown).

Correlations among performance measures, transfection, and DA depletion spaces

As observed earlier (Fig. 2f), larger and more dorsomedially placed DA depletions produced lower cued turning rates at baseline. In this experiment, higher TH-IR depletion scores also predicted greater $\mathrm{CNO}$-induced improvement in turning performance $\left(R^{2}=0.53, p=0.02\right)$. Moreover, the less overlap between TH-IR depletion spaces and transfection spaces (see Materials and Methods for quantifying overlaps), the greater were the CNO-induced improvements in performance $\left[R^{2}=\right.$ $0.79, p=0.004$; Fig. $7 f$, illustration of the TH-IR depletion and transfection areas of the two rats that showed the greatest (ranks 1 and 2; left) and smallest (ranks 9 and 10; right) CNO-induced improvement in performance]. As will be discussed below, stimulation of ChIs may have been more effective in cases where their reciprocal interactions with striatal DA activity were preserved.

\section{Discussion}

Falls in older people and persons with PD are a major cause of long-term hospitalization, nursing home admission, and rehabilitation (Baker and Harvey, 1985; Bloem et al., 2003; Grimbergen et al., 2004; Dellinger and Stevens, 2006). Although kinematic 

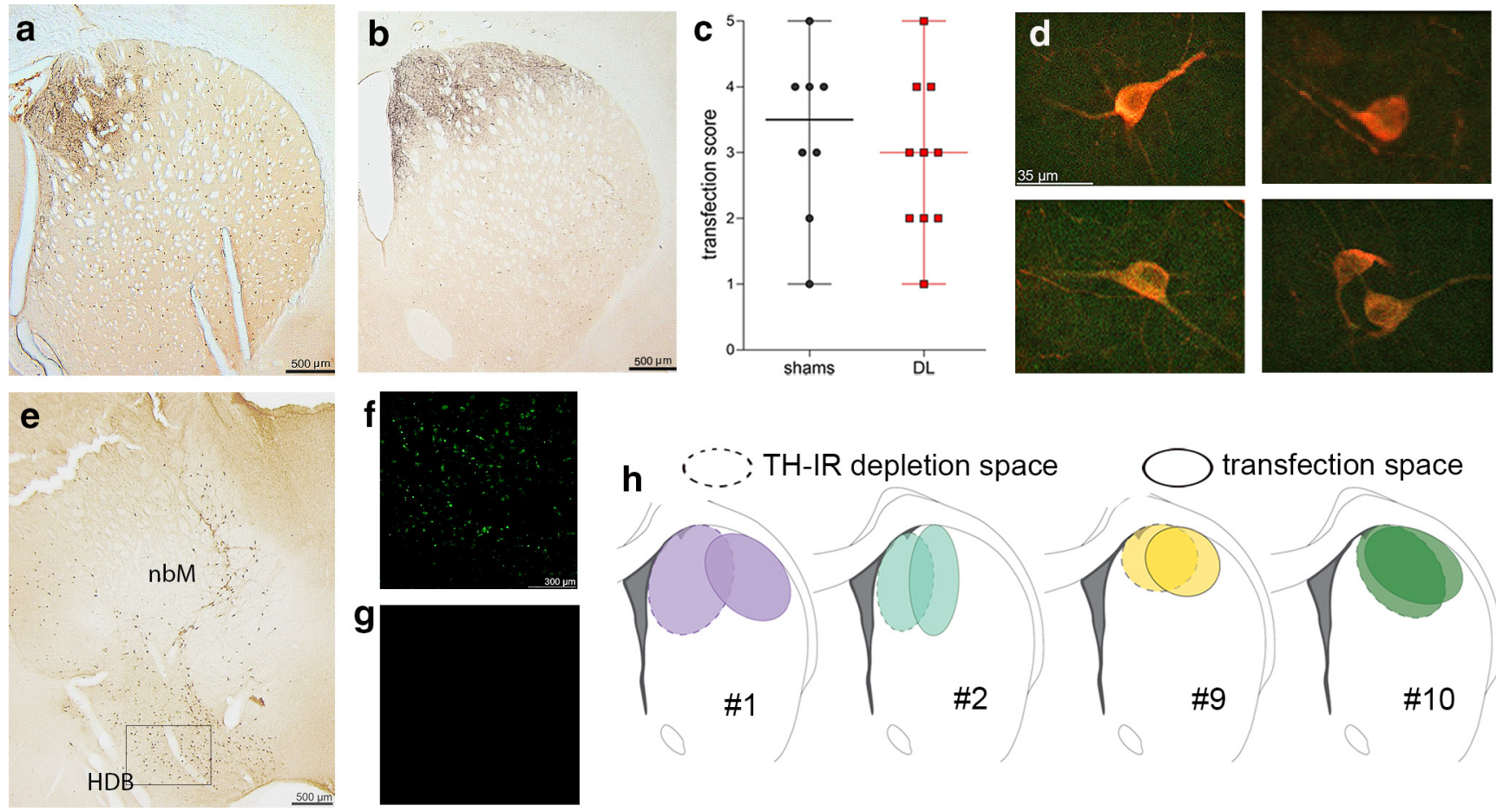

Figure 7. Viral transfection spaces, expression selectivity and relationship between histologic results and CNO-induced rescue of cued turning rates in DL rats. $\boldsymbol{a}, \boldsymbol{b}$, Representative images of coronal sections (magnification, 1.25×; scale bar, $500 \mu \mathrm{m}$ ) of striatal hM3Dq transfection spaces (chromogenic stains) from a sham-lesioned rat $(\boldsymbol{a})$ and a DL rat $(\boldsymbol{b})$. As already described, the transfection area was scored based on the size and localization in the prelimbic projection field of the dorsomedial striatum (spaces shown in $\boldsymbol{a}$ and $\boldsymbol{b}$ received a score of 4). c, Transfection space scores did not differ between sham-lesioned and DL rats, and they were also comparable to scores from intact rats expressing the inhibitory DREADD (Fig. 5f; for statistical analysis, see Results). Likewise, DL composite lesion scores did not differ from the DL scores obtained from rats from the experiment on (TTT performance (see Results; data not shown). $\boldsymbol{d}$, Examples of double-labeled (VAChT + mCherry) neurons in the dorsomedial striatum. Scale bar, $35 \mu \mathrm{m}$. These four examples were located within a TH-IR depleted space. The selectivity of expression of the viral construct by VAChT-positive neurons was not affected by dopamine depletion (for more details, see Results). $\boldsymbol{e}-\boldsymbol{g}$, Infusions of the viral construct into the dorsomedial striatum (see $\boldsymbol{a}, \boldsymbol{b}$ ) did not spread to the cholinergic neurons in the basal forebrain. e shows a coronal section of the basal forebrain (AP, $-1.1 \mathrm{~mm}$ from bregma) and chromogenically stained cholinergic neurons in the nucleus basalis of Meynert (nbM) and the horizontal nucleus of the diagonal band (HDB). Both basal forebrain subregions project to the cortex, with the latter predominately to prefrontal and visual regions (Luiten et al., 1987). $\boldsymbol{f}$ shows VAChT-positive, Alexa Fluor 488-labeled neurons in the HDB of a rat that received an infusion of the hM3Dq construct into the dorsomedial striatum. mCherry-positive fluorescence was virtually absent in the basal forebrain of rats [example in $\boldsymbol{g}$; scale bar, $\boldsymbol{f}($ for $\boldsymbol{f}, \boldsymbol{g}), 300 \mu \mathrm{m}$ ], indicating that, as expected, striatal transfection spaces did not extend to the cholinergic basal forebrain. $\boldsymbol{h}$, The TH-IR depletion spaces (broken contour lines) and transfection spaces (solid lines) of the two rats with the highest (ranks 1 and 2; left) and lowest (ranks 9 and 10; right) improvement in turning rates following the administration of CNO. Regardless of the degree of cholinergic losses and viral transfection spaces, TH depletions that were relatively more restricted to the dorsomedial striatum were associated with relatively lower performance at baseline (vehicle condition). Transfection spaces situated lateral to TH-IR depletions supported a relatively greater efficacy of $\mathrm{Chl}$ activation than viral expression spaces that were situated largely within TH-IR depleted regions.

variables, such as aberrant head motions and turning deficiencies, contribute to falls (Cole et al., 2010, 2011; Paul et al., 2014; Weaver et al., 2016; Oh et al., 2018), cognitive, specifically attentional, impairments and underlying cholinergic losses have been associated with a heightened propensity for falls (YogevSeligmann et al., 2008; Allcock et al., 2009; Bohnen et al., 2009b; LaPointe et al., 2010; Lord et al., 2010; Bohnen and Albin, 2011; O'Halloran et al., 2011; Plotnik et al., 2011; Yarnall et al., 2011). We previously demonstrated that rats with combined cortical cholinergic and striatal DA losses (DL rats) exhibit gait and postural abnormalities and high fall rates when tested using the MCMCT (Kucinski et al., 2013, 2015, 2017; Sarter et al., 2014; Kucinski and Sarter, 2015; Koshy Cherian et al., 2019).

Here, we first validated a new task, the CTTT, to broaden and specify the behavioral analysis of disruption of cholinergic-DA, cognitive-motor interactions in rodents. This task revealed impaired cued turning in DL rats. Spontaneous turning behavior and cued stopping remained unaffected. Thus, DL rats model the turning deficiencies seen in Parkinsonian fallers (Stack and Ashburn, 2008; Cheng et al., 2014). We then used both tasks to test the hypothesis that ChIs are a key element in circuitry integrating the corticostriatal processing of movement cues into movement control (Pisani et al., 2007; Deffains and Bergman,
2015; Tanimura et al., 2018). Chemogenetic inhibition of ChIs in otherwise intact rats markedly reduced cued turning rates and caused falls. These results support the hypothesis, derived from PET studies in Parkinsonian fallers (Bohnen et al., 2019), that ChI dysregulation is a mediator of impairments in gait and balance. Furthermore, in DL rats, stimulation of ChIs reduced fall rates, and nearly fully restored turning rates.

DL rats, a model of Parkinsonian falls, as well as intact rats expressing the inhibitory DREADDs and treated with CNO, failed to execute cued turns. Their ability to turn, per se, at the latest on treadmill restart in the opposite direction, and spontaneously in the open field, was not affected by the inhibition of ChIs. Thus, failures to execute cued turns may have reflected specifically an impaired utilization of the turn cue. Similar to our prior observation that falls in DL rats were positively correlated with striatal TH-IR depletion scores, but not the degree of cortical cholinergic deafferentation (Kucinski et al., 2013), cued turn rates were correlated with the extent of striatal DA depletion. Both findings suggest that cholinergic losses revealed or unmasked the impact of striatal DA losses on complex movement control. Because of their loss of cholinergic inputs to cortex and the resulting impairment in the cortical processing of turn cues, the corticostriatal transfer of information about such cues 

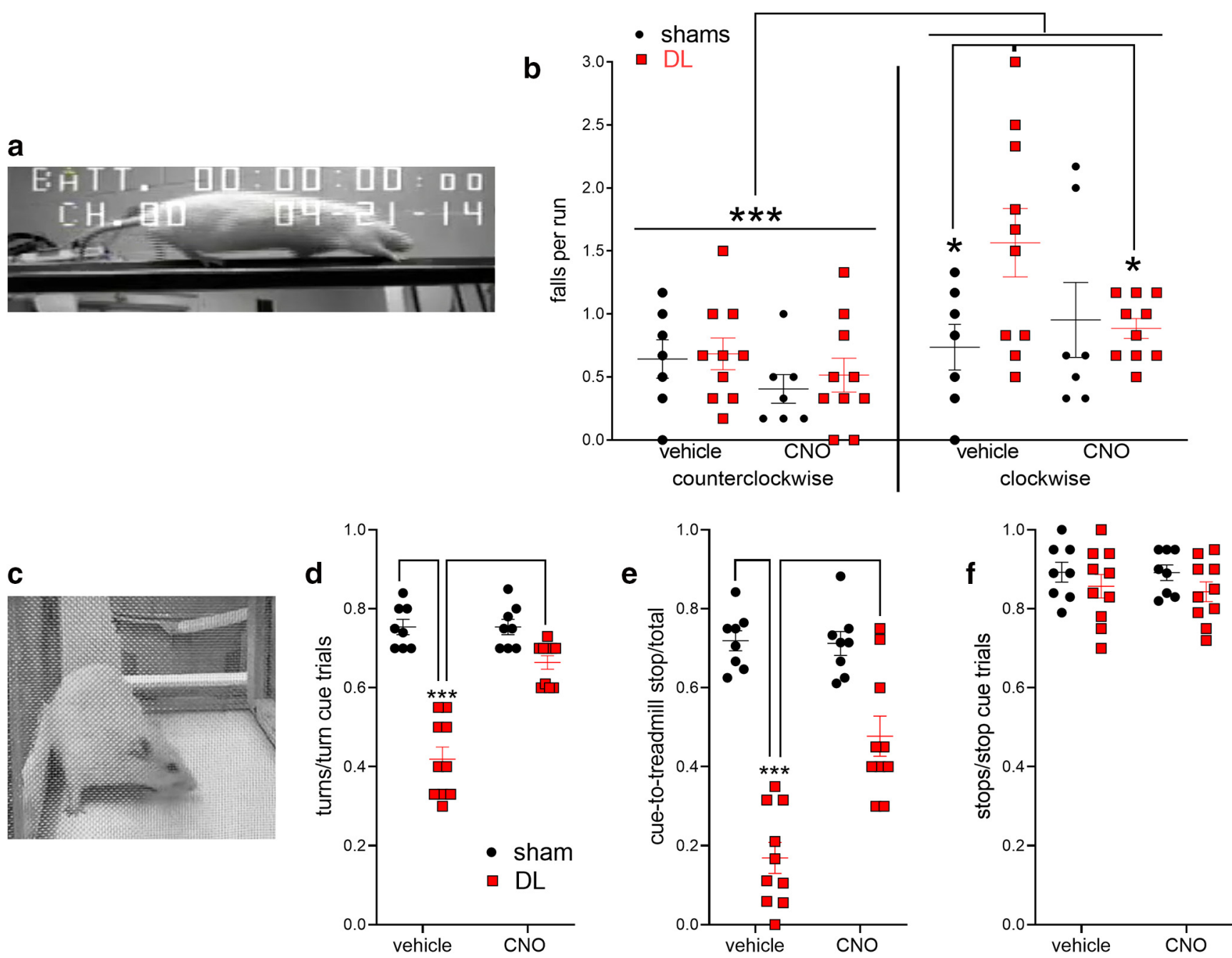

Figure 8. Effects of CNO-induced activation of an excitatory DREADD, expressed by Chls of sham-lesioned ( $n=7-8$ per task) and DL rats ( $n=10$ for both tasks; all graphs show individual data, means, and SEMs). $\boldsymbol{a}, \boldsymbol{b}$, Falls during traversal of the rotating rod were generally higher when the rod was rotated in the less familiar cw direction than when rotated cc. In the analysis of cw falls, vehicle-treated DL rats fell more often than sham-lesioned rats, and administration of CNO reduced fall rates in DL rats. c-e, In the analysis of cued turns (c) on the CTTT, significant interactions between lesion status and drug reflected that DL rats failed more often than sham-lesioned controls to turn on the presentation of the turn cue, and that the administration of CNO improved cued turn rates in DL rats $(\boldsymbol{d}, \boldsymbol{e})$. $\boldsymbol{f}$, Cued stops were unaffected by lesion status and CNO administration. CNO had no effects in a separate group of DL rats ( $n=8)$ expressing the viral control construct (see Results; data not shown).

may have been disrupted. In interaction with striatal DA losses, the weakened representation of the turn cue yielded a disruption of cue-guided striatal action selection (Kim et al., 2013; Hart et al., 2018b) and thus the capacity to execute cued turns. In trials beginning with a stop cue, rats typically stopped in response to the actual treadmill stop and thus may have used primarily proprioceptive information for stopping. Such information appears to have been spared from disruption by dual cholinergic-DA losses or inhibition of ChIs.

The findings that the inhibition of ChIs reproduced the cued turning deficits and, to a less complete degree, the high fall rates seen in DL rats agree with the hypothesis that these neurons are positioned to integrate corticostriatal input into striatal functioning, specifically in situations requiring cue-guided modification of behavior (Aoki et al., 2015). Indeed, as DA losses result in attenuated ChI function (McKinley et al., 2019), it is conceivable that, in DL rats, attenuated ChI function was an essential mediator of their cued turning deficits. Moreover, because the inhibition of ChIs not only disrupts the selection of corticostriatal input for further processing (Augustin et al., 2018; Aceves Buendia et al., 2019), but would also be expected to suppress the regulation of striatal DA (Threlfell et al., 2012; Brimblecombe et al., 2018), the effects of dual cholinergic-dopaminergic losses may have been essentially based on disruption of the reciprocal interactions among ChIs, corticostriatal activity, and nigrostriatal activity. These considerations suggest that impairments in gait and balance that are associated with $\mathrm{ChI}$ loss in aged persons and those with PD (Albin et al., 2018; Bohnen et al., 2019) may significantly worsen as accelerating cholinergic losses increasingly interact with striatal DA losses (Bohnen et al., 2009a,b, 2019).

The finding that the effects of ChI inhibition on falls in the MCMCT were less pronounced than the effects on cued turning behavior may reflect a relatively greater role of proprioceptive information in maintaining balance and movement when traversing highly dynamic surfaces when compared with cued turning in the CTTT. Such information reaches the striatum via diverse pathways, relying less critically on the frontostriatal loop than is the case for cued turning (Konczak et al., 2009; MacKinnon, 2018; Surgent et al., 2019). This hypothesis also explains the finding that $\mathrm{ChI}$ stimulation was relatively more effective in rescuing cued turning deficits than the high fall rates of DL rats. 
The results of the analysis of relatively more effective transfection spaces indicated that the stimulation of ChIs in intact striatal tissue was more effective than in cases in which transfected ChIs (partly) overlapped with DA-depleted areas. This observation is consistent with the view that effective ChI stimulation involves the activation of DA signaling (Threlfell et al., 2012; Kosillo et al., 2016; Brimblecombe et al., 2018), and thus ChI stimulation would be expected-as was the case-to be less effective in the absence of dopaminergic afferents. Consistent with the vast dendritic and axonal fields of ChIs (Kawaguchi et al., 1995) and their broad and coordinated responsivity to sensory stimuli (Schulz and Reynolds, 2013), the exact location of ChIs in the dorsal striatum for producing beneficial effects on stimulation may be relatively less important than the degree to which the afferent organization of the transfection space remains intact.

ChIs have been widely described as exhibiting autonomous, tonic firing patterns that are followed by large pauses (Wilson et al., 1990; Aosaki et al., 1995; Schulz and Reynolds, 2013; Tanimura et al., 2018). Chemogenetic suppression of both features of ChI activity likely contributed to the disruption of complex movement control assessed in our two tasks. However, because chemogenetic stimulation in vitro caused marked increases in ChI firing rates but also abolished pauses (AldrinKirk et al., 2018; Aoki et al., 2018), the finding that the stimulation of ChIs rescued the performance of DL rats cannot readily be mapped onto effects on canonical ChI firing characteristics. The determination of the neurophysiological correlates of the behavioral effects of chemogenetic manipulations of ChI activity awaits recordings in rats performing the CTTT.

The present results indicate an essential role of ChIs for complex movement control and demonstrate the feasibility of investigating the neuronal mechanisms underlying complex cognitivemotor interactions in rodent models. A consideration of the therapeutic potential of stimulation of ChIs to improve complex movement control in PD and reduce the risk for falls needs to be tempered by the possibility that such stimulation may not benefit the disease-defining motor symptoms of the disease (Maurice et al., 2015) and may be less effective in interaction with severe basal ganglia DA losses. A better understanding of the functions of the different components of ChI activity during complex movements, and of the impact of DA losses and diminished corticostriatal transfer of information about movement cues on ChI activity patterns, may yield neuronally and behaviorally specific strategies to enhance or rescue $\mathrm{ChI}$ function.

\section{References}

Aceves Buendia JJ, Tiroshi L, Chiu WH, Goldberg JA (2019) Selective remodeling of glutamatergic transmission to striatal cholinergic interneurons after dopamine depletion. Eur J Neurosci 49:824-833.

Agostinelli LJ, Geerling JC, Scammell TE (2019) Basal forebrain subcortical projections. Brain Struct Funct 224:1097-1117.

Albin RL, Bohnen NI, Muller M, Dauer WT, Sarter M, Frey KA, Koeppe RA (2018) Regional vesicular acetylcholine transporter distribution in human brain: a $\left[{ }^{18} \mathrm{~F}\right]$ fluoroethoxybenzovesamicol positron emission tomography study. J Comp Neurol 526:2884-2897.

Aldrin-Kirk P, Heuer A, Rylander Ottosson D, Davidsson M, Mattsson B, Björklund T (2018) Chemogenetic modulation of cholinergic interneurons reveals their regulating role on the direct and indirect output pathways from the striatum. Neurobiol Dis 109:148-162.

Allcock LM, Rowan EN, Steen IN, Wesnes K, Kenny RA, Burn DJ (2009) Impaired attention predicts falling in Parkinson's disease. Parkinsonism Relat Disord 15:110-115.

Amboni M, Barone P, Hausdorff JM (2013) Cognitive contributions to gait and falls: evidence and implications. Mov Disord 28:1520-1533.
Aoki S, Liu AW, Zucca A, Zucca S, Wickens JR (2015) Role of striatal cholinergic interneurons in set-shifting in the rat. J Neurosci 35:9424-9431.

Aoki S, Liu AW, Akamine Y, Zucca A, Zucca S, Wickens JR (2018) Cholinergic interneurons in the rat striatum modulate substitution of habits. Eur J Neurosci 47:1194-1205.

Aosaki T, Kimura M, Graybiel AM (1995) Temporal and spatial characteristics of tonically active neurons of the primate's striatum. J Neurophysiol 73:1234-1252.

Apicella P (2017) The role of the intrinsic cholinergic system of the striatum: what have we learned from TAN recordings in behaving animals? Neuroscience 360:81-94.

Armbruster BN, Li X, Pausch MH, Herlitze S, Roth BL (2007) Evolving the lock to fit the key to create a family of $\mathrm{G}$ protein-coupled receptors potently activated by an inert ligand. Proc Natl Acad Sci U S A 104:51635168 .

Arnold JC, Salvatore MF (2014) Getting to compliance in forced exercise in rodents: a critical standard to evaluate exercise impact in aging-related disorders and disease. J Vis Exp. Advance online publication. Retrieved August 22, 2014. doi: 10.3791/51827.

Augustin SM, Chancey JH, Lovinger DM (2018) Dual dopaminergic regulation of corticostriatal plasticity by cholinergic interneurons and indirect pathway medium spiny neurons. Cell Rep 24:2883-2893.

Baker SP, Harvey AH (1985) Fall injuries in the elderly. Clin Geriatr Med 1:501-512.

Berry AS, Sarter M, Lustig C (2017) Distinct frontoparietal networks underlying attentional effort and cognitive control. J Cogn Neurosci 29:12121225.

Bloem BR, Steijns JA, Smits-Engelsman BC (2003) An update on falls. Curr Opin Neurol 16:15-26.

Bohnen NI, Albin RL (2011) The cholinergic system and Parkinson disease. Behav Brain Res 221:564-573.

Bohnen NI, Muller ML, Kuwabara H, Cham R, Constantine GM, Studenski SA (2009a) Age-associated striatal dopaminergic denervation and falls in community-dwelling subjects. J Rehabil Res Dev 46:1045-1052.

Bohnen NI, Müller M, Koeppe RA, Studenski SA, Kilbourn MA, Frey KA, Albin RL (2009b) History of falls in Parkinson disease is associated with reduced cholinergic activity. Neurology 73:1670-1676.

Bohnen NI, Müller ML, Kotagal V, Koeppe RA, Kilbourn MR, Gilman S, Albin RL, Frey KA (2012) Heterogeneity of cholinergic denervation in Parkinson's disease without dementia. J Cereb Blood Flow Metab 32:1609-1617.

Bohnen NI, Frey KA, Studenski S, Kotagal V, Koeppe RA, Scott PJH, Albin RL, Müller M (2013) Gait speed in Parkinson disease correlates with cholinergic degeneration. Neurology 81:1611-1616.

Bohnen NI, Kanel P, Zhou Z, Koeppe RA, Frey KA, Dauer WT, Albin RL, Müller M (2019) Cholinergic system changes of falls and freezing of gait in Parkinson's disease. Ann Neurol 85:538-549.

Breese GR, Traylor TD (1971) Depletion of brain noradrenaline and dopamine by 6-hydroxydopamine. Br J Pharmacol 42:88-99.

Brimblecombe KR, Threlfell S, Dautan D, Kosillo P, Mena-Segovia J, Cragg SJ (2018) Targeted activation of cholinergic interneurons accounts for the modulation of dopamine by striatal nicotinic receptors. eNeuro 5: ENEURO.0397-17.2018.

Brown LA, Sleik RJ, Polych MA, Gage WH (2002) Is the prioritization of postural control altered in conditions of postural threat in younger and older adults? J Gerontol A Biol Sci Med Sci 57:M785-792.

Burk JA, Sarter M (2001) Dissociation between the attentional functions mediated via basal forebrain cholinergic and GABAergic neurons. Neuroscience 105:899-909.

Burk JA, Herzog CD, Porter MC, Sarter M (2002) Interactions between aging and cortical cholinergic deafferentation on attention. Neurobiol Aging 23:467-477.

Castelhano-Carlos MJ, Baumans V (2009) The impact of light, noise, cage cleaning and in-house transport on welfare and stress of laboratory rats. Lab Anim 43:311-327.

Cheng FY, Yang YR, Wang CJ, Wu YR, Cheng SJ, Wang HC, Wang RY (2014) Factors influencing turning and its relationship with falls in individuals with Parkinson's disease. PLoS One 9:e93572.

Chuhma N, Mingote S, Moore H, Rayport S (2014) Dopamine neurons control striatal cholinergic neurons via regionally heterogeneous dopamine and glutamate signaling. Neuron 81:901-912. 
Cohen J (1988) Statistical power analysis for the behavioral sciences, Ed 2. Hillsdale, NJ: Erlbaum.

Cole MH, Silburn PA, Wood JM, Worringham CJ, Kerr GK (2010) Falls in Parkinson's disease: kinematic evidence for impaired head and trunk control. Mov Disord 25:2369-2378.

Cole MH, Silburn PA, Wood JM, Kerr GK (2011) Falls in Parkinson's disease: evidence for altered stepping strategies on compliant surfaces. Parkinsonism Relat Disord 17:610-616.

Dalley JW, Theobald DE, Bouger P, Chudasama Y, Cardinal RN, Robbins TW (2004) Cortical cholinergic function and deficits in visual attentional performance in rats following $192 \mathrm{IgG}$-saporin-induced lesions of the medial prefrontal cortex. Cereb Cortex 14:922-932.

Deffains M, Bergman H (2015) Striatal cholinergic interneurons and corticostriatal synaptic plasticity in health and disease. Mov Disord 30:10141025.

Dellinger AM, Stevens JA (2006) The injury problem among older adults: mortality, morbidity and costs. J Safety Res 37:519-522.

Doig NM, Magill PJ, Apicella P, Bolam JP, Sharott A (2014) Cortical and thalamic excitation mediate the multiphasic responses of striatal cholinergic interneurons to motivationally salient stimuli. J Neurosci 34:3101-3117.

Doumas M, Smolders C, Krampe RT (2008) Task prioritization in aging: effects of sensory information on concurrent posture and memory performance. Exp Brain Res 187:275-281.

Emmons EB, De Corte BJ, Kim Y, Parker KL, Matell MS, Narayanan NS (2017) Rodent medial frontal control of temporal processing in the dorsomedial striatum. J Neurosci 37:8718-8733.

Gemperle AY, McAllister KH, Olpe HR (2003) Differential effects of iloperidone, clozapine, and haloperidol on working memory of rats in the delayed non-matching-to-position paradigm. Psychopharmacology (Berl) 169:354-364.

Gilat M, Shine JM, Walton CC, O'Callaghan C, Hall JM, Lewis SJG (2015) Brain activation underlying turning in Parkinson's disease patients with and without freezing of gait: a virtual reality fMRI study. NPJ Parkinsons Dis 1:15020.

Gomez JL, Bonaventura J, Lesniak W, Mathews WB, Sysa-Shah P, Rodriguez LA, Ellis RJ, Richie CT, Harvey BK, Dannals RF, Pomper MG, Bonci A, Michaelides M (2017) Chemogenetics revealed: DREADD occupancy and activation via converted clozapine. Science 357:503-507.

Greenwald AG, Gonzalez R, Harris RJ, Guthrie D (1996) Effect sizes and p values: what should be reported and what should be replicated? Psychophysiology 33:175-183.

Grimbergen YA, Munneke M, Bloem BR (2004) Falls in Parkinson's disease. Curr Opin Neurol 17:405-415.

Gritton HJ, Howe WM, Mallory CS, Hetrick VL, Berke JD, Sarter M (2016) Cortical cholinergic signaling controls the detection of cues. Proc Natl Acad Sci U S A 113:E1089-E1097.

Gritton HJ, Howe WM, Romano MF, DiFeliceantonio AG, Kramer MA, Saligrama V, Bucklin ME, Zemel D, Han X (2019) Unique contributions of parvalbumin and cholinergic interneurons in organizing striatal networks during movement. Nat Neurosci 22:586-597.

Guo Q, Wang D, He X, Feng Q, Lin R, Xu F, Fu L, Luo M (2015) Wholebrain mapping of inputs to projection neurons and cholinergic interneurons in the dorsal striatum. PLoS One 10:e0123381.

Guthrie M, Leblois A, Garenne A, Boraud T (2013) Interaction between cognitive and motor cortico-basal ganglia loops during decision making: a computational study. J Neurophysiol 109:3025-3040.

Hart G, Bradfield LA, Balleine BW (2018a) Prefrontal corticostriatal disconnection blocks the acquisition of goal-directed action. J Neurosci 38:1311-1322.

Hart G, Bradfield LA, Fok SY, Chieng B, Balleine BW (2018b) The bilateral prefronto-striatal pathway is necessary for learning new goal-directed actions. Curr Biol 28:2218-2229.e7.

Howe WM, Gritton HJ, Lusk NA, Roberts EA, Hetrick VL, Berke JD, Sarter M (2017) Acetylcholine release in prefrontal cortex promotes gamma oscillations and theta-gamma coupling during cue detection. J Neurosci 37:3215-3230.

Kawaguchi Y, Wilson CJ, Augood SJ, Emson PC (1995) Striatal interneurones: chemical, physiological and morphological characterization. Trends Neurosci 18:527-535.

Kim H, Lee D, Jung MW (2013) Signals for previous goal choice persist in the dorsomedial, but not dorsolateral striatum of rats. J Neurosci 33:5263 .
Kim K, Müller M, Bohnen NI, Sarter M, Lustig C (2019) The cortical cholinergic system contributes to the top-down control of distraction: evidence from patients with Parkinson's disease. Neuroimage 190:107-117.

Klug JR, Engelhardt MD, Cadman CN, Li H, Smith JB, Ayala S, Williams EW, Hoffman H, Jin X (2018) Differential inputs to striatal cholinergic and parvalbumin interneurons imply functional distinctions. Elife 7 : e35657.

Konczak J, Corcos DM, Horak F, Poizner H, Shapiro M, Tuite P, Volkmann J, Maschke M (2009) Proprioception and motor control in Parkinson's disease. J Mot Behav 41:543-552.

Koshy Cherian A, Kucinski A, Wu R, de Jong IEM, Sarter M (2019) Co-treatment with rivastigmine and idalopirdine reduces the propensity for falls in a rat model of falls in Parkinson's disease. Psychopharmacology (Berl) 236:1701-1715.

Kosillo P, Zhang YF, Threlfell S, Cragg SJ (2016) Cortical control of striatal dopamine transmission via striatal cholinergic interneurons. Cereb Cortex 26:4160-4169.

Kucinski A, Sarter M (2015) Modeling Parkinson's disease falls associated with brainstem cholinergic systems decline. Behav Neurosci 129:96-104.

Kucinski A, Paolone G, Bradshaw M, Albin R, Sarter M (2013) Modeling fall propensity in Parkinson's disease: deficits in the attentional control of complex movements in rats with cortical-cholinergic and striatal-dopaminergic deafferentation. J Neurosci 33:16522-16539.

Kucinski A, Albin RL, Lustig C, Sarter M (2015) Modeling falls in Parkinson's disease: slow gait, freezing episodes and falls in rats with extensive striatal dopamine loss. Behav Brain Res 282:155-164.

Kucinski A, de Jong IE, Sarter M (2017) Reducing falls in Parkinson's disease: interactions between donepezil and the 5-HT6 receptor antagonist idalopirdine on falls in a rat model of impaired cognitive control of complex movements. Eur J Neurosci 45:217-231.

Kucinski A, Lustig C, Sarter M (2018) Addiction vulnerability trait impacts complex movement control: evidence from sign-trackers. Behav Brain Res 350:139-148.

Kucinski A, Kim Y, Sarter M (2019) Basal forebrain chemogenetic inhibition disrupts the superior complex movement control of goal-tracking rats. Behav Neurosci 133:121-134.

Kucinski A, Phillips KB, Koshy Cherian A, Sarter M (2020) Rescuing the attentional performance of rats with cholinergic losses by the M1 positive allosteric modulator TAK-071. Psychopharmacology (Berl)) 237:137153

Landis JR, Koch GG (1977) The measurement of observer agreement for categorical data. Biometrics 33:159-174.

LaPointe LL, Stierwalt JAG, Maitland CG (2010) Talking while walking: cognitive loading and injurious falls in Parkinson's disease. Int J Speech Lang Pathol 12:455-459.

Lord S, Rochester L, Hetherington V, Allcock LM, Burn D (2010) Executive dysfunction and attention contribute to gait interference in "off" state Parkinson's disease. Gait Posture 31:169-174.

Luiten PG, Gaykema RP, Traber J, Spencer DG Jr (1987) Cortical projection patterns of magnocellular basal nucleus subdivisions as revealed by anterogradely transported Phaseolus vulgaris leucoagglutinin. Brain Res 413:229-250.

MacKinnon CD (2018) Sensorimotor anatomy of gait, balance, and falls. Handb Clin Neurol 159:3-26.

MacLaren DA, Browne RW, Shaw JK, Krishnan Radhakrishnan S, Khare P, España RA, Clark SD (2016) Clozapine N-Oxide administration produces behavioral effects in Long-Evans rats: implications for designing DREADD experiments. eNeuro 3:ENEURO.0219-16.2016.

Mahler SV, Aston-Jones G (2018) CNO evil? Considerations for the use of DREADDs in behavioral neuroscience. Neuropsychopharmacology 43:934-936.

Mailly P, Aliane V, Groenewegen HJ, Haber SN, Deniau JM (2013) The rat prefrontostriatal system analyzed in 3D: evidence for multiple interacting functional units. J Neurosci 33:5718-5727.

Mamaligas AA, Barcomb K, Ford CP (2019) Cholinergic transmission at muscarinic synapses in the striatum is driven equally by cortical and thalamic inputs. Cell Rep 28:1003-1014.e3.

Martinez V, Sarter M (2008) Detection of the moderately beneficial cognitive effects of low-dose treatment with haloperidol or clozapine in an animal model of the attentional impairments of schizophrenia. Neuropsychopharmacology 33:2635-2647. 
Maurice N, Liberge M, Jaouen F, Ztaou S, Hanini M, Camon J, Deisseroth K, Amalric M, Kerkerian-Le Goff L, Beurrier C (2015) Striatal cholinergic interneurons control motor behavior and basal ganglia function in experimental parkinsonism. Cell Rep 13:657-666.

McGaughy J, Sarter M (1998) Sustained attention performance in rats with intracortical infusions of 192 IgG-saporin-induced cortical cholinergic deafferentation: effects of physostigmine and FG 7142. Behav Neurosci 112:1519-1525.

McGaughy J, Kaiser T, Sarter M (1996) Behavioral vigilance following infusions of 192 IgG-saporin into the basal forebrain: selectivity of the behavioral impairment and relation to cortical AChE-positive fiber density. Behav Neurosci 110:247-265.

McKinley JW, Shi Z, Kawikova I, Hur M, Bamford IJ, Sudarsana Devi SP, Vahedipour A, Darvas M, Bamford NS (2019) Dopamine deficiency reduces striatal cholinergic interneuron function in models of Parkinson's disease. Neuron 103:1056-1072. e1056.

Mersmann F, Bohm S, Bierbaum S, Dietrich R, Arampatzis A (2013) Young and old adults prioritize dynamic stability control following gait perturbations when performing a concurrent cognitive task. Gait Posture 37:373-377.

Montero-Odasso M, Verghese J, Beauchet O, Hausdorff JM (2012) Gait and cognition: a complementary approach to understanding brain function and the risk of falling. J Am Geriatr Soc 60:2127-2136.

Müller ML, Albin RL, Kotagal V, Koeppe RA, Scott PJ, Frey KA, Bohnen NI (2013) Thalamic cholinergic innervation and postural sensory integration function in Parkinson's disease. Brain 136:3282-3289.

Nagamatsu LS, Munkacsy M, Liu-Ambrose T, Handy TC (2013) Altered visual-spatial attention to task-irrelevant information is associated with falls risk in older adults. Neuropsychologia 51:3025-3032.

O’Halloran AM, Pénard N, Galli A, Fan CW, Robertson IH, Kenny RA (2011) Falls and falls efficacy: the role of sustained attention in older adults. BMC Geriatr 11:85.

Oh K, Stanley CJ, Damiano DL, Kim J, Yoon J, Park HS (2018) Biomechanical evaluation of virtual reality-based turning on a self-paced linear treadmill. Gait Posture 65:157-162.

Okada K, Nishizawa K, Setogawa S, Hashimoto K, Kobayashi K (2018) Taskdependent function of striatal cholinergic interneurons in behavioural flexibility. Eur J Neurosci 47:1174-1183.

Parada MA, Hernandez L, P, de Parada M, Rada P, Murzi E (1997) Selective action of acute systemic clozapine on acetylcholine release in the rat prefrontal cortex by reference to the nucleus accumbens and striatum. J Pharmacol Exp Ther 281:582-588.

Paul SS, Sherrington C, Canning CG, Fung VS, Close JC, Lord SR (2014) The relative contribution of physical and cognitive fall risk factors in people with Parkinson's disease: a large prospective cohort study. Neurorehabil Neural Repair 28:282-290.

Pavcic J, Matjacic Z, Olensek A (2014) Kinematics of turning during walking over ground and on a rotating treadmill. J Neuroeng Rehabil 11:127.

Pisani A, Bernardi G, Ding J, Surmeier DJ (2007) Re-emergence of striatal cholinergic interneurons in movement disorders. Trends Neurosci 30:545-553.

Plotnik M, Giladi N, Dagan Y, Hausdorff JM (2011) Postural instability and fall risk in Parkinson's disease: impaired dual tasking, pacing, and bilateral coordination of gait during the "ON" medication state. Exp Brain Res 210:529-538.

Rochester L, Yarnall AJ, Baker MR, David RV, Lord S, Galna B, Burn DJ (2012) Cholinergic dysfunction contributes to gait disturbance in early Parkinson's disease. Brain 135:2779-2788.

Rodriguez A, Zhang HQ, Klaminder J, Brodin T, Andersson PL, Andersson M (2018) ToxTrac: a fast and robust software for tracking organisms. Methods Ecol Evol 9:460-464.

Roth BL (2016) DREADDs for Neuroscientists. Neuron 89:683-694.

Rueter LE, Ballard ME, Gallagher KB, Basso AM, Curzon P, Kohlhaas KL (2004) Chronic low dose risperidone and clozapine alleviate positive but not negative symptoms in the rat neonatal ventral hippocampal lesion model of schizophrenia. Psychopharmacology (Berl) 176:312-319.
Sarter M, Fritschy JM (2008) Reporting statistical methods and statistical results in EJN. Eur J Neurosci 28:2363-2364.

Sarter M, Lustig C (2020) Forebrain cholinergic signaling: wired and phasic, not tonic, and causing behavior. J Neurosci 40:712-719.

Sarter M, Albin RL, Kucinski A, Lustig C (2014) Where attention falls: increased risk of falls from the converging impact of cortical cholinergic and midbrain dopamine loss on striatal function. Exp Neurol 257:120 129.

Sarter M, Lustig C, Blakely RD, Koshy Cherian A (2016) Cholinergic genetics of visual attention: human and mouse choline transporter capacity variants influence distractibility. J Physiol Paris 110:10-18.

Schulz JM, Reynolds JN (2013) Pause and rebound: sensory control of cholinergic signaling in the striatum. Trends Neurosci 36:41-50.

Smith KS, Bucci DJ, Luikart BW, Mahler SV (2016) DREADDs: use and application in behavioral neuroscience. Behav Neurosci 130:137-155.

Stack E, Ashburn A (2008) Dysfunctional turning in Parkinson's disease. Disabil Rehabil 30:1222-1229.

Strafella AP, Ko JH, Grant J, Fraraccio M, Monchi O (2005) Corticostriatal functional interactions in Parkinson's disease: a rTMS/[11C]raclopride PET study. Eur J Neurosci 22:2946-2952.

St Peters M, Demeter E, Lustig C, Bruno JP, Sarter M (2011) Enhanced control of attention by stimulating mesolimbic-corticopetal cholinergic circuitry. J Neurosci 31:9760-9771.

Stuart S, Galna B, Delicato LS, Lord S, Rochester L (2017) Direct and indirect effects of attention and visual function on gait impairment in Parkinson's disease: influence of task and turning. Eur J Neurosci 46:1703-1716.

Surgent OJ, Dadalko OI, Pickett KA, Travers BG (2019) Balance and the brain: a review of structural brain correlates of postural balance and balance training in humans. Gait Posture 71:245-252.

Tago H, Kimura H, Maeda T (1986) Visualization of detailed acetylcholinesterase fiber and neuron staining in rat brain by a sensitive histochemical procedure. J Histochem Cytochem 34:1431-1438.

Tanimura A, Pancani T, Lim SAO, Tubert C, Melendez AE, Shen W, Surmeier DJ (2018) Striatal cholinergic interneurons and Parkinson's disease. Eur J Neurosci 47:1148-1158.

Terry AV Jr, Hill WD, Parikh V, Waller JL, Evans DR, Mahadik SP (2003) Differential effects of haloperidol, risperidone, and clozapine exposure on cholinergic markers and spatial learning performance in rats. Neuropsychopharmacology 28:300-309.

Threlfell S, Lalic T, Platt NJ, Jennings KA, Deisseroth K, Cragg SJ (2012) Striatal dopamine release Is triggered by synchronized activity in cholinergic interneurons. Neuron 75:58-64.

Turner JG, Parrish JL, Hughes LF, Toth LA, Caspary DM (2005) Hearing in laboratory animals: strain differences and nonauditory effects of noise. Comp Med 55:12-23.

Virmani T, Pillai L, Glover A, Doerhoff SM, Williams DK, Garcia-Rill E, Larson-Prior L (2018) Impaired step-length setting prior to turning in Parkinson's disease patients with freezing of gait. Mov Disord 33:18231825.

Weaver TB, Robinovitch SN, Laing AC, Yang Y (2016) Falls and Parkinson's disease: evidence from video recordings of actual fall events. J Am Geriatr Soc 64:96-101.

Wilson CJ, Chang HT, Kitai ST (1990) Firing patterns and synaptic potentials of identified giant aspiny interneurons in the rat neostriatum. J Neurosci 10:508-519.

Yarnall A, Rochester L, Burn DJ (2011) The interplay of cholinergic function, attention, and falls in Parkinson's disease. Mov Disord 26:2496-2503.

Yogev-Seligmann G, Hausdorff JM, Giladi N (2008) The role of executive function and attention in gait. Mov Disord 23:329-342.

Yogev-Seligmann G, Hausdorff JM, Giladi N (2012) Do we always prioritize balance when walking? Towards an integrated model of task prioritization. Mov Disord 27:765-770

Zucca S, Zucca A, Nakano T, Aoki S, Wickens J (2018) Pauses in cholinergic interneuron firing exert an inhibitory control on striatal output in vivo. Elife 7:e32510 